\title{
Prognostic Significance of Apoptosis Regulators in B-Cell Chronic Lymphocytic Leukemia
}

\author{
Ahmad Baraka1, Shereen El Shorbagy², Ola M. Elfarargy2*, Rasha Haggag2, \\ Lobna A. Abdelaziz ${ }^{3}$, Salah F. Elsayed ${ }^{4}$, Khaled A. Elbana ${ }^{4}$ \\ ${ }^{1}$ Clinical Pathology Department, Faculty of Medicine, Zagazig University, Zagazig, Egypt \\ ${ }^{2}$ Medical Oncology Department, Faculty of Medicine, Zagazig University, Zagazig, Egypt \\ ${ }^{3}$ Clinical Oncology \& Nuclear Medicine Department, Faculty of Medicine, Zagazig University, Zagazig, Egypt \\ ${ }^{4}$ Internal Medicine Department, Faculty of Medicine, Zagazig University, Zagazig, Egypt \\ Email: *elfarargyola@yahoo.com
}

How to cite this paper: Baraka, A., El Shorbagy, S., Elfarargy, O.M., Haggag, R., Abdelaziz, L.A., Elsayed, S.F. and Elbana, K.A. (2017) Prognostic Significance of Apoptosis Regulators in B-Cell Chronic Lymphocytic Leukemia. Journal of Cancer Therapy, 8, 360-385.

https://doi.org/10.4236/jct.2017.84032

Received: January 8, 2017

Accepted: April 25, 2017

Published: April 28, 2017

Copyright $\odot 2017$ by authors and Scientific Research Publishing Inc. This work is licensed under the Creative Commons Attribution International License (CC BY 4.0).

http://creativecommons.org/licenses/by/4.0/

\begin{abstract}
Background: High levels of MCL-1 and BCL-2 proteins have been found in Chronic Lymphocytic Leukemia (CLL), and inversely correlated with response to treatment. BCL-2/Bax ratio is the main director of apoptosis in CLL. The study aimed to clarify the prognostic role of MCL-1, BCL-2 and BCL-2/ Bax ratio in B-CLL. Patients \& method: Estimation of MCL-1, BCL-2 and Bax expressions by a flow cytometry in 45 B-CLL patients and the prognostic value of these markers were correlated with other well-known established prognostic markers and treatment response. Results: MCL-1 was expressed in $60 \%$ of cases while BCL- 2 was expressed in $82.2 \%$ of cases. MCL-1 expression was significantly high in male gender, short lymphocyte doubling time (LDT), and high expression of CD $38(p<0.001)$. High (Serum LDH, serum $\beta 2 \mathrm{M}$, CD38 expression), low ZAP-70 expression, splenomegaly and higher Rai stage were significantly increased in patients with high expression of BCL-2 $(p<$ 0.001 ); also a significant decrease in (HB level, platelet count), and increase in serum $\mathrm{LDH}$, serum $\beta 2 \mathrm{M}$, high C-D38 expression, low ZAP-70 expression, the poor cytogenetic and splenomegaly in patients with high expression of BCL-2/ Bax ratio $(p<0.001)$. Among the 39 patients who started treatment when indicated responding patients had statistically significant lower BCL-2/Bax ratio than non-responding patients, although their lower mean of MCL-1 and BCL2 expressions values were insignificant. In conclusion: MCL-1, BCL-2 expressions and BCL-2/Bax ratio could be useful potential predictive and prognostic markers in B-CLL.
\end{abstract}

\section{Keywords}

Myeloid Cell Leukemia 1, B-Cell Lymphoma 2, BAX, B-Cell Chronic Lymphocytic Leukemia 


\section{Introduction}

The most prevalent form of adult leukemia is B-Chronic lymphocytic leukemia (B-CLL); it is characterized by accumulation of CD5+ and CD23+ B-cell lymphocytes [1]. It is a heterogeneous disease with variable clinical course; some patients presented with an indolent disease and need no or little treatment, while others have aggressive diseases at diagnosis. Rai and Binet staging systems cannot predict the disease outcome in early stages of Chronic Lymphocytic Leukemia [2]. Additionally to known prognostic markers, other markers like $\mathrm{V}$ region genes mutation, genomic aberrations, and expression of CD38 are counted as significant prognostic markers [3]. Although response can be attained in $60 \%$ of patients by existing treatment, the disease is still not curable [4]. This incurability is mainly caused by CD5+ B cells accumulation which is responsible for cell immortality [5]. Recently, it is recommended that survival signals' modulation interferes with apoptosis which possibly plays the vital tool in CLL pathogenesis. BCL-2 (B-cell lymphoma-2) family of anti-apoptotic (BCL-xl, BCL-2, MCL-1 and BCL-w) and proapoptotic (bok, bax, and bak) proteins are essential for apoptosis control in CLL [6]. BCL-2 claimed as the major protein in CLL which helps for survival prediction [7]. Some studies showed that high BCL-2 levels and low expression of bax protein are connected to treatment resistant in CLL cells [8]. Numeroustrials established that in CLL; the equilibrium between members of pro- and anti-apoptotic BCL-2 family impacts the sensitivity to chemotherapy and patients survival [9]; also it is found that this balance between anti- and pro-apoptotic proteins is more important than intensity of each protein expression [10]. BCL-2/Bax ratio was identified as the main determinant in regulation of apoptosis in CLL by some investigators [11] [12], while others denied this [13]. In our study, we evaluated the effect of MCL-1, BCL-2, and $\mathrm{BCL}-2 / \mathrm{Bax}$ ratio on tumor response, survival, and relation to other established markers in CLL.

\section{Method}

\subsection{Subjects and Methods}

We enrolled 45 denovo B-CLL patients, who were presented to Clinical pathology, Medical Oncology, Clinical oncology and Internal Medicine departments, Zagazig University Hospitals. In the period between June 2013 and May 2015, complete history taking was done for all patients, clinical examination, and laboratory investigations; $\mathrm{CBC}$, direct Coombs' test, $\mathrm{LDH}$ and $\beta 2 \mathrm{M}$ estimation.

An international scoring system based on expression patterns of immunophenotyping markers in CLL has been established. Patients with Chronic B lymphoproliferative disorders were diagnosed by evidence of persistent absolute lymphocytosis $>5000 / \mu \mathrm{l}\left(5 \times 10^{9} /\right.$ liter $)$ for 3 months. Immunophenotyping was performed by monoclonal antibodies panels: CD3, CD7, CD5, CD19, CD20, CD22, $\mathrm{K} / \lambda$, CD23, CD79b, and FMC7, also estimation of cytoplasmic ZAP-70 expression and surface CD38 [14]. Anti-ZAP-70-FITC (Becton-Dickinson, USA and Bi- 
oscience) with an intrastain kit (Dako) and anti-CD38-PE (DAKO, CA, USA) were provided with a permeabilizing agent. For FITC, PC-5 and PE (negative control) conjugated monoclonal antibody specific isotypic controls were used. We selected lymphocytes in the forward scatter against side scatter dot blot and gated as CD19/CD5 positive cells. Samples analysis was performed by multicolor FCM (FACS Caliber flow cytometry Becton Dickinson, USA). The percentage of gated cells was measured as positive expression over the corresponding isotypic control with cut-off $\geq 30 \%$ for CD38 and $\geq 20 \%$ for ZAP- 70 .

\subsection{Special Investigations}

Flow cytometric analysis of MCL-1, BCL-2 and Bax expressions by using FITCconjugated MoAb (Dako, Glostrup, Denmark). Peripheral blood samples (EDTA blood) were tested initially with diagnosis or before any treatment. All samples were initially incubated with CD19 PE and CD5 APC MoAbs for 30 min at $4^{\circ} \mathrm{C}$. Subsequently the cells after washing twice in PBS were fixed and permeabilized with Cell Permeabilizationkit. Samples were then incubated at $4^{\circ} \mathrm{C}$ for $30 \mathrm{~min}$ with $10 \mathrm{uL}$ anti-MCL-1, anti-BCL-2, and anti BAX FITC conjugated. We evaluated MCL-1, BCL-2 and Baxas relative mean fluorescence intensities (rMFIs). The ratio between BCL-2 and Bax (rMFIs), and non-specific MoAb MFI on B cells also were calculated. In current study, a cut-off of $25 \%$ of positive cells was chosen to determine MCL-1 positive in CLL cases [15] and a cut-off of $10 \%$ of positive cells was chosen to discriminate BCL-2 and BAX positive from negative in CLL cases [16].

Figure 1(a) shows a flowcytometric histogram for positive markers CD23, CD19, CD22 with co-expression between CD5and CD20 with K/L showed dim expression, and also shows that; MCL-1, BCL-2 and CD38 are positive. Figure 1 (b) shows a flow cytometric histogram for positive markers CD23, CD19, CD22 with co-expression between CD5and CD20; this is a case of B-CLL. And also shows that MCL-1, BCL-2 and CD38 are negative. Separation of two subgroups with different OS and/or PFS was done depending on BCL-2/Bax-2 ratio, probabilities was calculated by different methods like Youden's index, receiver-operating characteristic (ROC) analysis and median values. We concluded BCL-2/Bax median value $\geq 1.50$ (range $0.27-6.10$ ) as the threshold value and this was confirmed by ROC analysis Online Supplementary, Figure 2.

\subsection{Cytogenetic Analysis}

By using fluorescence in situ hybridization (FISH) technique, Cytogenetic analysis was done on peripheral blood, a locus specific identifier DNA probe (LSI) Kit was used, Vysis (Abbott Park, Ill, USA).

\subsection{When to Initiate Therapy}

There are indications to begin treatment for CLL patients including: elevated total leucocytes count with a lymphocytes doubling time $<12$ months, having anemia or thrombocytopenia as a result of bone marrow infiltration, development 

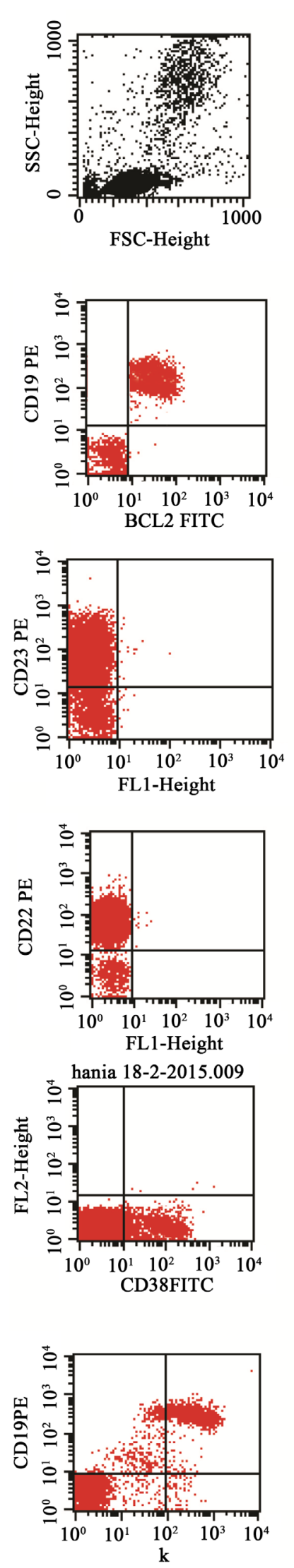
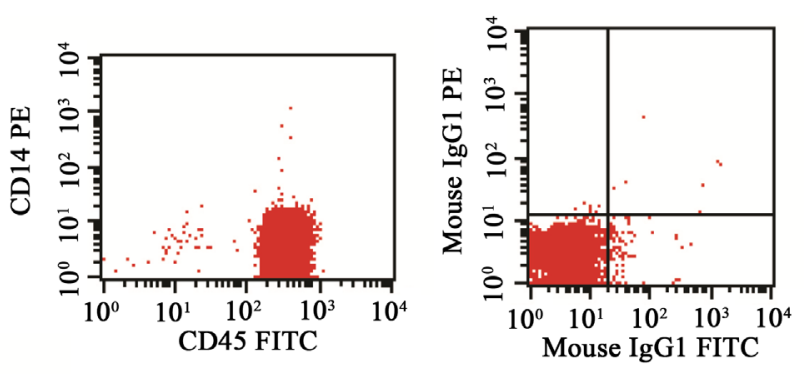

\section{Tubu: BCL2/CD19}

Quad Location: 8, 12

Quad \% Gated \% Total

$\begin{array}{crr}\text { UL } & 0.30 & 0.22 \\ \text { UR } & 90.11 & 67.17\end{array}$

$\begin{array}{llr}\text { LL } & 9.46 \quad 7.05\end{array}$

LR $0.13 \quad 0.10$

Tubu: CD23

Quad Location: 9, 12

Quad \% Gated \% Total

$\begin{array}{lrr}\text { UL } & 84.34 & 67.22 \\ \text { UR } & 0.28 & 0.22 \\ \text { LL } & 15.23 & 12.14 \\ \text { LR } & 0.15 & 0.12\end{array}$

Tubu: CD22

Quad Location: 9, 12

Quad \% Gated \% Total

$\begin{array}{lll}\text { UL } & 92.55 & 75.83\end{array}$

$\begin{array}{lll}\text { UR } & 0.15 \quad 0.12\end{array}$

LL $\quad 7.30 \quad 5.98$

$\begin{array}{lll}\text { LR } & 0.00 \quad 0.00\end{array}$

Tubu: CD38

Quad Location: 11, 13

Quad \% Gated \% Total

$\begin{array}{rrr}\text { UL } & 0.00 & 0.00 \\ \text { UR } & 0.08 & 0.06 \\ \text { LL } & 91.43 & 72.99 \\ \text { LR } & 38.49 & 36.78\end{array}$

Tubu: K/L/CD19PE

Quad Location: 94, 8

Quad \% Gated \% Total

$\begin{array}{lll}\text { UR } & 35.10 & 12.83\end{array}$

LL $\quad 51.22 \quad 18.72$

LR $\quad 1.75 \quad 0.64$
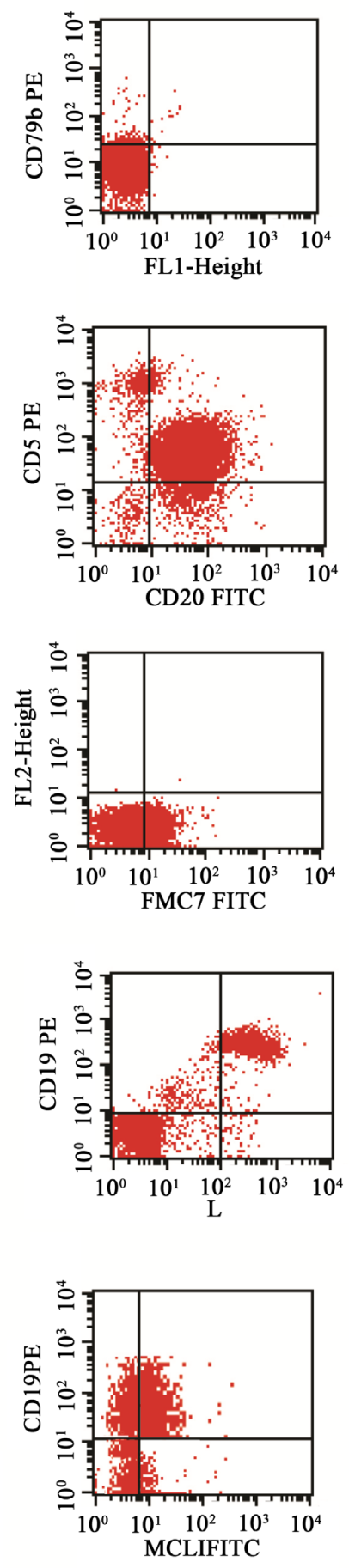

Tubu: CD79B

Quad Location: 8, 21

Quad \% Gated \% Total

$\begin{array}{lrr}\text { UL } & 6.03 & 4.75 \\ \text { UR } & 0.18 & 0.14 \\ \text { LL } & 93.62 & 73.72 \\ \text { LR } & 0.17 & 0.13\end{array}$

\section{Tubu: CD20/CD5}

Quad Location: 9, 12

Quad \% Gated \% Total

$\begin{array}{rrr}\text { UL } & 5.46 & 4.34 \\ \text { UR } & 84.02 & 66.83 \\ \text { LL } & 2.20 & 1.75 \\ \text { LR } & 8.32 & 6.62\end{array}$

Tubu: FMC7

Quad Location: 9, 12

Quad \% Gated \% Total

$\begin{array}{lrr}\text { UL } & 0.01 & 0.01 \\ \text { UR } & 0.02 & 0.02 \\ \text { LL } & 67.91 & 54.37 \\ \text { LR } & 32.05 & 25.66\end{array}$

Tubu: L/CD19PE

Quad Location: 94, 8

Quad \% Gated \% Total

$\begin{array}{lrr}\text { UL } & 8.89 & 3.25 \\ \text { UR } & 10.00 & 5.94 \\ \text { LL } & 51.49 & 18.82 \\ \text { LR } & 1.48 & 0.54\end{array}$

Tubu: MCL1 FITC/CD19PE

Quad Location: 6, 11

Quad \% Gated \% Total

$\begin{array}{lrr}\text { UL } & 20.08 & 8.01 \\ \text { UR } & 70.65 & 50.08 \\ \text { LL } & 9.74 & 1.19 \\ \text { LR } & 10.53 & 2.94\end{array}$

(a) 

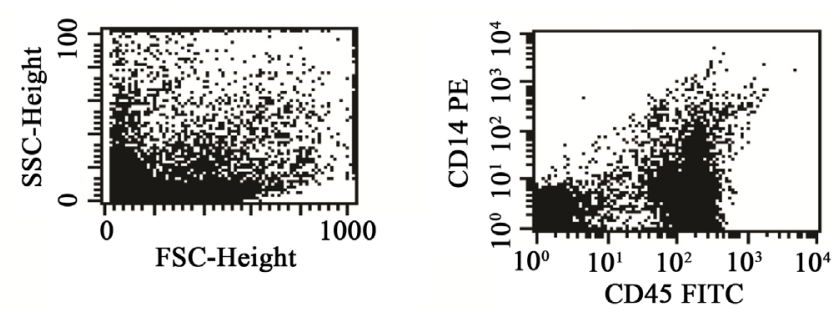
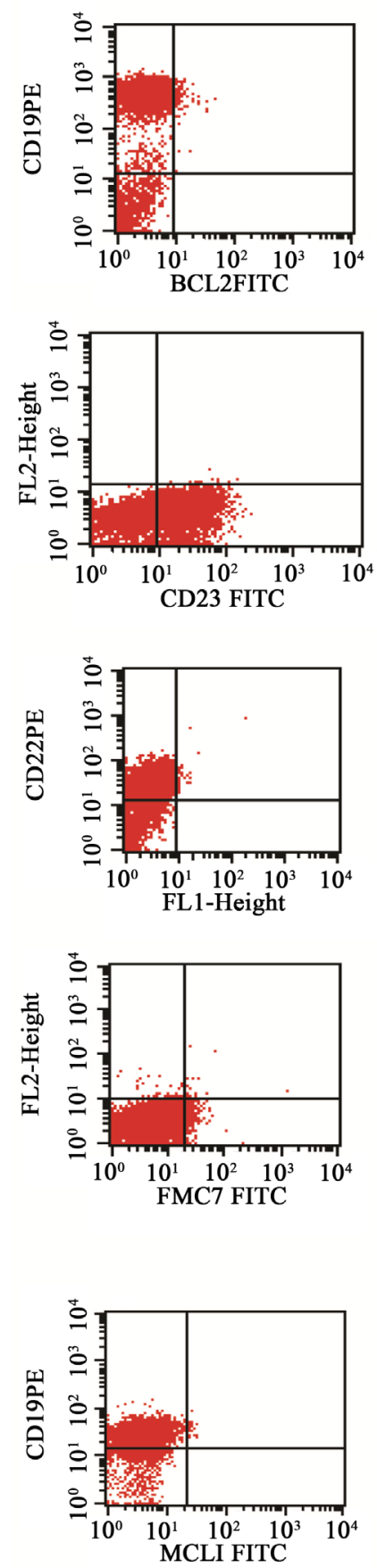

Tubu: FMC7

Quad Location: 19, 9

\begin{tabular}{rrr} 
Quad & \% Gated & \% Total \\
\hline UL & 1.97 & 1.10 \\
UR & 0.65 & 0.36 \\
LL & 93.40 & 52.11 \\
LR & 3.98 & 2.22
\end{tabular}

Tubu: MCL1 FITC/CD19PE

Quad Location: 21, 13

\begin{tabular}{crr} 
Quad & \% Gated & \% Total \\
\hline UL & 88.36 & 82.89 \\
UR & 0.26 & 0.24 \\
LL & 11.38 & 10.68 \\
LR & 0.00 & 0.00
\end{tabular}

Tubu: BCL2/CD19

Quad Location: 9, 12

\begin{tabular}{rrr} 
Quad $\%$ Gated \% Total \\
\hline UL & 79.02 & 40.49
\end{tabular}

$\begin{array}{lrr}\text { UR } & 2.73 & 1.40\end{array}$

LL $\quad 18.23 \quad 9.34$

$\begin{array}{lll}\text { LR } & 0.02 \quad 0.01\end{array}$

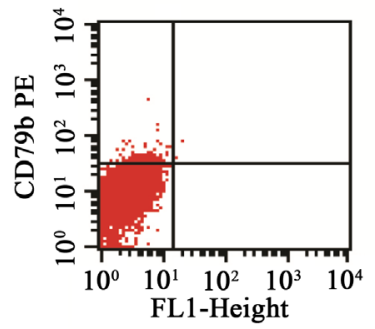

Tubu: CD79B

Quad Location: 14, 27

Quad \% Gated \% Total

$\begin{array}{ccc}\text { UL } & 4.21 & 2.38 \\ \text { UR } & 0.07 & 0.04\end{array}$

LL $\quad 95.71 \quad 54.15$

$\begin{array}{lll}\text { LR } & 0.02 & 0.01\end{array}$

Tubu: CD20/CD5

Quad Location: 9, 12

Quad Location: 9, 12

\begin{tabular}{crr} 
Quad & $\%$ Gated & $\%$ Total \\
\hline UL & 0.05 & 0.03 \\
UR & 0.88 & 0.50 \\
LL & 33.09 & 18.76 \\
LR & 65.97 & 37.40
\end{tabular}

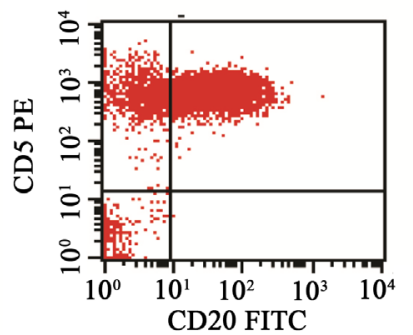

\begin{tabular}{rrr} 
Quad & $\%$ Gated & $\%$ Total \\
\hline UL & 19.45 & 11.01
\end{tabular}

UR $\quad 75.79 \quad 42.91$

LL $\quad 4.75 \quad 2.69$

$\begin{array}{lll}\text { LR } & 0.02 \quad 0.01\end{array}$

Tubu: CD22

Quad Location: 9, 12

\begin{tabular}{rrr} 
Quad & $\%$ Gated & $\%$ Total \\
\hline UL & 76.05 & 39.73
\end{tabular}

$\begin{array}{lll}\text { UR } & 0.94 & 0.49\end{array}$

LL $\quad 23.01 \quad 12.02$

LR $\quad 0.00 \quad 0.00$

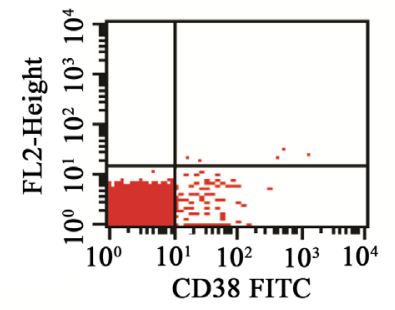

Tubu: CD38

Quad Location: 11, 13

\begin{tabular}{rrr} 
Quad & \% Gated & \% Total \\
\hline UL & 0.00 & 0.00 \\
UR & 0.08 & 0.06 \\
LL & 91.43 & 72.99 \\
LR & 8.49 & 6.78
\end{tabular}

(b)

Figure 1. (a) Flow cytometric dot plots of BCL-2, MCL1 and bax on CD19+ cells on peripheral blood sample of one negative representative CLL case with reported MFI (mean fluorescent intensity) values; (b) Flow cytometric dot plots of BCL-2, MCL1 and bax on CD19+ cells on peripheral blood sample of one positive representative CLL case with reported MFI values. 


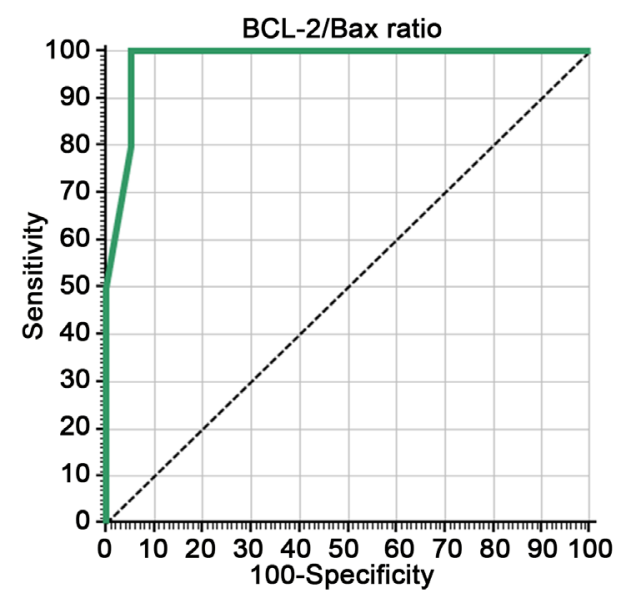

Figure 2. Receiver operating characteristic (ROC) curve of BCL-2/Bax ratio as a predictor of overall response of CLL patients to treatment.

of systemic B-symptoms, bulky lymphadenopathy, increasing organomegally, and recurrent infection. For patients $<60$ years fludarabine/cyclophos-phamide + Rituximab (FCR) protocol was given, and for CLL patients $>60$ years chlorambucil + Rituximab were given. Complete and partial remission of the disease were measured as overall response, while failure of response was defined as stable (did not achieve any remission) or progressive disease. To determine when to start first treatment with early stages disease and to evaluate the disease outcome we followed up patients up to 18 months. Six months post start of treatment; re-evaluation was done for our patients for response assessment according to guidelines of National Cancer Institute-sponsored group [17].

\section{Statistical Analysis}

Continuous variables were expressed as the mean \pm SD \& median (range), and the categorical variables were expressed as a number (percentage). Continuous variables were checked for normality by using Shapiro-Wilk test. Mann Whitney $\mathrm{U}$ test was used to compare between two groups of non-normally distributed variables. Kruskal Wallis $\mathrm{H}$ test was used to compare between more than two groups of normally distributed variables. Percent of categorical variables were compared using the Pearson's Chi-square test or Fisher's exact test when was appropriate. Trend of change in distribution of relative frequencies between ordinal data were compared using Chi-square test for trend. Receiver operating characteristic (ROC) curve analysis was used to identify optimal cut-off values of BCL-2/Bax ratio with maximum sensitivity and specificity for prediction of overall response of CLL patients to treatment. Area under Curve (AUROC) was also calculated, criteria to qualify for AUC were as follows: $0.90-1=$ excellent, $0.80-0.90=$ good, 0.70 $0.80=$ fair; $0.60-0.70=$ poor; and $0.50-0.6=$ fail. The optimal cutoff point was established at point of maximum accuracy. Strength of relationship between time to start first treatment and flow-cytometry markers were determined by computing Spearman's correlation coefficient, $(+)$ sign was indicating direct relationship \& (-) sign was indicator for inverse relationship was indicated by (-) sign, 
values near to 0 was indicator for weak relationship \& values near 1 was indicating strong relationship. All tests were two sided. A significant $\mathrm{p}$-value was $<0.05$. SPSS 22.0 for windows (SPSS Inc., Chicago, IL, USA) and MedCalc windows (MedCalc Software bvba 13, Ostend, Belgium) was used in performing all statistics.

\section{Results}

The clinical, laboratory, and outcome of all B-CLL patients are summarized in Table 1. Forty five patients with B-CLL were included; 26 males and 19 females, their age ranged between ( $38-74)$ years, with a mean value of $56.3 \pm 12.3$. Twenty patients presented with low stages (0-II) according to Rai staging, and followed up until any indication to start treatment occurred, while 25 patients presented with high stages (III-IV), and began treatment once they diagnosed. MCL-1 was expressed in $60 \%$ of B-CLL cases with mean \pm SD (60.6 \pm 41.98$)$, MCL-1 expression was significantly high in male patients, short lymphocytic doubling time (LDT), and high expression of CD $38(p<0.001)$ as shown in Table 2, BCL-2 was expressed in $82.2 \%$ of B-CLL cases, with mean \pm SD $(52.93+29.05)$. There was statistically significant increase in (serum LDH, serum $\beta 2 \mathrm{M}, \mathrm{CD} 38$ ), low ZAP-70 expression, splenomegaly and higher Rai stage in patients with high expression of BCL-2 compared to those with low expression $(p<0.001)$, Table 3.

\subsection{ROC Analysis}

To establish the clinical significance of BCL-2/Bax ratio, we calculated the index of test validity for responders to treatment $(\mathrm{CR}+\mathrm{PR})$ and non-responders (NR). The best results of sensitivity and specificity were obtained at the cut-off point of 1.6. Table 4 and Figure 2 show ROC curve of BCL-2/Bax ratio as a predictor of overall response of CLL patients to treatment.

There was statistically significant decrease in (HB level, platelet count), and increase in serum $\mathrm{LDH}$, serum $\beta 2 \mathrm{M}$, high CD38 expression, low ZAP-70 expression, poor cytogenetic and splenomegaly in patients with high expression of BCL-2/Bax ratio compared to those with low ratio $(p<0.001)$, Table 5 .

\subsection{The Relation of Apoptosis Regulators and Rai Staging System}

We classified our patients according to Rai staging system, and divided them into two groups; low risk group (stage 0-II) and high risk group (stage III-IV). There were significant associations between BCL-2, BCL-2/Bax ratio, and Rai staging system $(p<0.05)$, while MCL-1 expression has no relation to Rai system $(p>0.05)$.

\subsection{Response to Treatment Table 6}

Thirty-nine patients $(86.6 \%)$ received chemotherapy during the follow-up period, and demonstrated variable response; 8 patients achieved CR, 12 achieved $\mathrm{PR}$, and 19 were non-responders (NR) to treatment. Six patients did not require starting treatment for CLL, and one patient lost follow-up. 
Table 1. Clinical, laboratory, and outcome of all B-CLL patients.

\begin{tabular}{|c|c|c|c|c|c|}
\hline $\begin{array}{l}\text { Characteristics } \\
\text { Age (year) }\end{array}$ & No. & $(\%)$ & $\begin{array}{c}\text { Characteristics } \\
\text { Cytogenetic Analysis }\end{array}$ & No. & (\%) \\
\hline Mean \pm SD & 56.31 & \pm 12.3 & Normal & 20 & $(44.4 \%)$ \\
\hline Median (Range) & 54 & $(38-74)$ & del 13 & 4 & $(8.9 \%)$ \\
\hline$\leq 60$ years & 25 & $(55.6 \%)$ & del 11 & 5 & $(11.1 \%)$ \\
\hline$>60$ years & 20 & $(44.4 \%)$ & Trisomy 12 & 6 & $(13.3 \%)$ \\
\hline Sex & & & del 17 & 5 & $(11.1 \%)$ \\
\hline $\begin{array}{l}\text { Male } \\
\text { Female }\end{array}$ & & $\begin{array}{l}26(57.8 \%) \\
19(42.2 \%)\end{array}$ & Complex & 5 & $(11.1 \%)$ \\
\hline & & & CD19 (\%) & & \\
\hline Rai classification & & & Mean \pm SD & 82.92 & \pm 15.67 \\
\hline Stage $0,1 \& 2$ & 20 & $(44.4 \%)$ & Median (Range) & 89.10 & $(32.84-97.61)$ \\
\hline Stage $3 \& 4$ & 25 & $(55.6 \%)$ & CD23 (\%) & & \\
\hline Clinical mainfestaion & & & Mean \pm SD & 69.40 & \pm 27.74 \\
\hline Hepatomegally & 22 & $(48.9 \%)$ & Median (Range) & 79.27 & $(0.38-95.74)$ \\
\hline Lymphadenopathy & 31 & $(68.9 \%)$ & CD5/CD20 (\%) & & \\
\hline Spleenomegally & 33 & $(73.3 \%)$ & Mean \pm SD & 73.22 & \pm 23.52 \\
\hline WBCs $\left(\times 10^{3} / \mathrm{mm}^{3}\right)$ & & & Median (Range) & 79.90 & $(8.41-96.02)$ \\
\hline Mean \pm SD & 46.80 & \pm 31.71 & CD79b (\%) & & \\
\hline Median (Range) & 31.60 & $(17.50-122.8)$ & Mean \pm SD & 57.18 & \pm 33.93 \\
\hline$<100 \times 10^{3} / \mathrm{mm}^{3}$ & 39 & $(86.7 \%)$ & Median (Range) & 66.57 & $(1.93-95.51)$ \\
\hline$\geq 100 \times 10^{3} / \mathrm{mm}^{3}$ & 6 & $(13.3 \%)$ & FMc7 (\%) & & \\
\hline Absolute lymphocytes $\left(\times 10^{3} / \mathrm{mm}^{3}\right)$ & & & Mean \pm SD & 17.01 & \pm 24.89 \\
\hline Mean \pm SD & 26.27 & \pm 16.42 & Median (Range) & 4.85 & $(0.06-88.44)$ \\
\hline Median (Range) & 18 & $(9.10-67)$ & CD22 (\%) & & \\
\hline$<30 \times 10^{3} / \mathrm{mm}^{3}$ & 33 & $(73.3 \%)$ & Mean \pm SD & 24.95 & \pm 14.39 \\
\hline$\geq 30 \times 10^{3} / \mathrm{mm}^{3}$ & 12 & $(26.7 \%)$ & Median (Range) & 20.79 & $(7.12-70.69)$ \\
\hline Platelet count $\left(\times 10^{3} / \mathrm{mm}^{3}\right)$ & & & CD38 (\%) & & \\
\hline Mean \pm SD & 121.13 & \pm 47.20 & Mean \pm SD & 23.79 & \pm 25.41 \\
\hline Median (Range) & 111 & $(45-205)$ & Median (Range) & 8 & $(0.30-91.59)$ \\
\hline$<100 \times 10^{3} / \mathrm{mm}^{3}$ & 19 & $(42.2 \%)$ & $<30 \%$ & 28 & $(62.2 \%)$ \\
\hline$\geq 100 \times 10^{3} / \mathrm{mm}^{3}$ & 26 & $(57.8 \%)$ & $>30 \%$ & 17 & $(37.8 \%)$ \\
\hline Hemoglobin (g/dl) & & & MCL-1 (\%) & & \\
\hline Mean \pm SD & 10.73 & \pm 2.29 & Mean \pm SD & 60.60 & \pm 41.98 \\
\hline Median (Range) & 10.20 & $(6.60-14.30)$ & Median (Range) & 91.30 & $(0.60-99.70)$ \\
\hline$<12 \mathrm{~g} / \mathrm{dl}$ & 25 & $(55.6 \%)$ & $<25 \%$ & 18 & $(40 \%)$ \\
\hline$\geq 12 \mathrm{~g} / \mathrm{dl}$ & 20 & $(44.4 \%)$ & $>25 \%$ & 27 & $(60 \%)$ \\
\hline LDH (U/L) & & & BCL-2 (\%) & & \\
\hline
\end{tabular}




\section{Continued}

$\begin{array}{cccccc}\text { Mean } \pm \text { SD } & 354 & \pm 107.94 & \text { Mean } \pm \text { SD } & 52.20 & \pm 29.24 \\ \text { Median (Range) } & 340 & (190-540) & \text { Median (Range) } & 53.90 & (2-93.20) \\ \leq 350 \mathrm{U} / \mathrm{L} & 23 & (51.1 \%) & <10 \% & 8 & (17.8 \%) \\ >350 \mathrm{U} / \mathrm{L} & 22 & (48.9 \%) & >10 \% & 37 & (82.2 \%)\end{array}$

B2-microglobulin (mg/L)

\begin{tabular}{|c|c|c|c|c|c|}
\hline Mean \pm SD & 2.91 & \pm 0.99 & $<20 \%$ & 27 & $(60 \%)$ \\
\hline Median (Range) & 3 & $(1.40-5)$ & $>20 \%$ & 18 & $(40 \%)$ \\
\hline$<3.5 \mathrm{mg} / \mathrm{L}$ & 29 & $(64.4 \%)$ & Time to begin ttt (months) for all $\mathrm{Pt}$ & & \\
\hline$\geq 3.5 \mathrm{mg} / \mathrm{L}$ & 16 & $(35.6 \%)$ & Mean \pm SD & 5.07 & \pm 4.23 \\
\hline Coomb's test & & & Median (Range) & 7 & $(2-14)$ \\
\hline Negative & 38 & $(84.4 \%)$ & Response $^{*}$ & & \\
\hline Positive & 7 & $(15.6 \%)$ & & & \\
\hline cytic doubling time (LDT) & & & No response (NR) & 19 & $(42.2 \%)$ \\
\hline$<12$ months & 21 & $(46.7 \%)$ & Overall response (ORR) & 20 & $(44.5 \%)$ \\
\hline$>12$ months & 24 & $(53.3 \%)$ & & & \\
\hline
\end{tabular}

Continuous variables were expressed as mean \pm SD \& median (range); categorical variables were expressed as number (percentage). ${ }^{\star} 6$ patients $(13.3 \%)$ were not received treatment.

Table 2. Relation between clinicopathological features, flow cytometry markers and MCL-1 expression.

\begin{tabular}{|c|c|c|c|c|c|c|c|}
\hline \multirow{3}{*}{ Characteristics } & \multirow{2}{*}{\multicolumn{2}{|c|}{$\begin{array}{c}\text { All } \\
(\mathrm{N}=45)\end{array}$}} & \multicolumn{4}{|c|}{ MCL-1 } & \multirow{3}{*}{$p$-value } \\
\hline & & & \multicolumn{2}{|c|}{$<25 \%(\mathrm{~N}=18)$} & \multicolumn{2}{|c|}{$>25 \%(\mathrm{~N}=27)$} & \\
\hline & No. & (\%) & No. & $(\%)$ & No. & (\%) & \\
\hline \multicolumn{8}{|l|}{ Age (years) } \\
\hline Mean \pm SD & 56.31 & \pm 12.3 & 52.33 & \pm 11.77 & 58.96 & \pm 12.01 & \multirow[b]{2}{*}{$0.074 \bullet$} \\
\hline Median (Range) & 54 & $(38-74)$ & 50.50 & $(38-73)$ & 61 & $(42-74)$ & \\
\hline$\leq 60$ years & 25 & $(55.6 \%)$ & 13 & $(52 \%)$ & 12 & $(48 \%)$ & \multirow{2}{*}{0.066} \\
\hline$>60$ years & 20 & $(44.4 \%)$ & 5 & $(25 \%)$ & 15 & $(75 \%)$ & \\
\hline \multicolumn{8}{|l|}{ Sex } \\
\hline Male & 26 & $(57.8 \%)$ & 14 & $(53.8 \%)$ & 12 & $(46.2 \%)$ & \multirow[b]{2}{*}{0.027} \\
\hline Female & 19 & $(42.2 \%)$ & 4 & $(21.1 \%)$ & 15 & $(78.9 \%)$ & \\
\hline \multicolumn{8}{|l|}{ Rai classification } \\
\hline Stage $0,1 \& 2$ & 20 & $(44.4 \%)$ & 9 & $(45 \%)$ & 11 & $(55 \%)$ & \multirow{2}{*}{0.540} \\
\hline Stage $3 \& 4$ & 25 & $(55.6 \%)$ & 9 & $(36 \%)$ & 16 & $(64 \%)$ & \\
\hline \multicolumn{8}{|l|}{ Hepatomegally } \\
\hline Absent & 23 & $(51.1 \%)$ & 7 & $(30.4 \%)$ & 16 & $(69.6 \%)$ & \multirow{2}{*}{0.181} \\
\hline Present & 22 & $(48.9 \%)$ & 11 & $(50 \%)$ & 11 & (50\%) & \\
\hline
\end{tabular}




\section{Continued}

Lymphadenopathy

$\begin{array}{llllclll}\text { Absent } & 14 & (31.1 \%) & 7 & (50 \%) & 7 & (50 \%) & 0.357 \\ \text { Present } & 31 & (68.9 \%) & 11 & (35.5 \%) & 20 & (64.5 \%) & \end{array}$

Spleenomegally

$\begin{array}{llllllll}\text { Absent } & 12 & (26.7 \%) & 7 & (58.3 \%) & 5 & (41.7 \%) & 0.175 \\ \text { Present } & 33 & (73.3 \%) & 11 & (33.3 \%) & 22 & (66.7 \%) & \end{array}$

WBCs $\left(\times 10^{3} / \mathrm{mm}^{3}\right)$

Mean $\pm \mathrm{SD}$
Median (Range)
$<100 \times 10^{3} / \mathrm{mm}^{3}$
$\geq 100 \times 10^{3} / \mathrm{mm}^{3}$

$\begin{array}{ccccccc}46.80 & \pm 31.71 & 49.00 & \pm 38.39 & 45.33 & \pm 27.06 & 0.651 \bullet \\ 31.60 & (17.50-122.80) & 31 & (17.50-122.80) & 37.20 & (17.50-118.50) & \\ 39 & (86.7 \%) & 14 & (35.9 \%) & 25 & (64.1 \%) & \\ 6 & (13.3 \%) & 4 & (66.7 \%) & 2 & (33.3 \%) & 0.199\end{array}$

Absolute lymphocytes $\left(\times 10^{3} / \mathrm{mm}^{3}\right)$

Mean $\pm \mathrm{SD}$
Median (Range)
$<30 \times 10^{3} / \mathrm{mm}^{3}$
$\geq 30 \times 10^{3} / \mathrm{mm}^{3}$

$\begin{array}{ccccccr}26.27 & \pm 16.42 & 27.60 & \pm 21.73 & 25.39 & \pm 12.06 & 0.487 \bullet \\ 18 & (9.10-67) & 16 & (9.10-67) & 24 & (12.50-55.20) & \\ 33 & (73.3 \%) & 13 & (39.4 \%) & 20 & (60.6 \%) & \\ 12 & (26.7 \%) & 5 & (41.7 \%) & 7 & (58.3 \%) & \end{array}$

Platelet count $\left(\times 10^{3} / \mathrm{mm}^{3}\right)$

$$
\text { Mean } \pm \text { SD }
$$

Median (Range)

$<100 \times 10^{3} / \mathrm{mm}^{3}$

$\geq 100 \times 10^{3} / \mathrm{mm}^{3}$

Hemoglobin (g/dl)

Mean \pm SD
Median (Range)

$$
\begin{aligned}
& <12 \mathrm{~g} / \mathrm{dl} \\
& \geq 12 \mathrm{~g} / \mathrm{dl}
\end{aligned}
$$

LDH (U/L)

Mean \pm SD

Median (Range)

$\leq 350 \mathrm{U} / \mathrm{L}$

$>350 \mathrm{U} / \mathrm{L}$

B2-microglobulin (mg/L)

$$
\begin{gathered}
\text { Mean } \pm \mathrm{SD} \\
\text { Median (Range) } \\
<3.5 \mathrm{mg} / \mathrm{L} \\
\geq 3.5 \mathrm{mg} / \mathrm{L}
\end{gathered}
$$

$\begin{array}{cc}121.13 & \pm 47.20 \\ 111 & (45-205) \\ 19 & (42.2 \%) \\ 26 & (57.8 \%)\end{array}$

126.77

111.50

$\pm 45.62$

117.37

(65 - 205)

102

(31.6\%)

12

(46.2\%)

13

14

$\pm 48.71$

(45 - 200)

(68.4\%)

(53.8\%)

0.324

$\begin{array}{cc}10.73 & \pm 2.29 \\ 10.20 & (6.60-14.30)\end{array}$

10.81

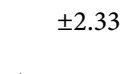

10.40

(7.60 - 14.30)

10.67

$\pm 2.31$

25

(55.6\%)

(44.4\%)

9

(36\%)

(45\%)

10.20

(6.60 - 14)

$$
9
$$

16

(64\%)

(55\%)

0.540

$$
11
$$$$
\text { (55\%) }
$$

0.935

$\begin{array}{ccccccc}354 & \pm 107.94 & 341.11 & \pm 106.70 & 362.59 & \pm 109.91 & 0.479 \bullet \\ 340 & (190-540) & 340 & (200-540) & 340 & (190-540) & \\ 23 & (51.1 \%) & 9 & (39.1 \%) & 14 & (60.9 \%) & \\ 22 & (48.9 \%) & 9 & (40.9 \%) & 13 & (59.1 \%) & 0.903\end{array}$




\section{Continued}

Coomb's test

Negative

Positive

38

7

21

24

$>12$ months

Cytogenetic analysis

$$
\begin{aligned}
& \text { Normal } \\
& \text { del } 13 \\
& \text { del } 11
\end{aligned}
$$

Trisomy 12

del 17

Complex

CD38 (\%)

Mean \pm SD

$$
\begin{aligned}
& <30 \% \\
& >30 \%
\end{aligned}
$$

BCL-2 (\%)

Mean \pm SD

Median (Range)

$$
\begin{aligned}
& <10 \% \\
& >10 \%
\end{aligned}
$$

ZAP-70

$$
\begin{aligned}
& <20 \% \\
& >20 \%
\end{aligned}
$$

BCL-2/Bax ratio

Mean \pm SD
Median (Range)
$\leq 1.6$

(84.4\%)

(15.6\%)

(46.7\%)

(53.3\%)

16

$(42.1 \%)$

(28.6\%)

22

(57.9\%)

(71.4\%)

0.684

12
6

(57.1\%)

9

(42.9\%)

(25\%)

18

(75\%)

0.028

$\begin{array}{ll}(44.4 \%) & 8 \\ (8.9 \%) & 3 \\ (11.1 \%) & 3 \\ (13.3 \%) & 3 \\ (11.1 \%) & 0 \\ (11.1 \%) & 1\end{array}$

(40\%)

\section{2}

(60\%)

(75\%) $\quad 1$

$$
(60 \%)
$$

(50\%)

2

(40\%)

(50\%)

$(0 \%)$

3

(100\%)

(20\%)

4

(80\%)

0.20

$0.431 \bullet$

$(0.30-91.57) \quad 32 \quad(0.60-91.59)$

0.003

$\begin{array}{lll}(11.8 \%) & 15 & (88.2 \%)\end{array}$

$\begin{array}{lll}(57.1 \%) & 12 & (42.9 \%) \\ (11.8 \%) & 15 & (88.2 \%)\end{array}$

27

18

$52.20 \quad \pm 29.24$

53.90

$(2-93.20)$

(17.8\%)

$(82.2 \%)$

(60\%)

(40\%)

\subsection{9}

$\pm 30.40$

53.90

$(3-93.20)$

(50\%)

(37.8\%)

52.61

$\pm 29.03$

4

48.20

(2 - 91.20)

$0.981 \bullet$

$0.694 \S$

(62.2\%)

(33.3\%)

18

(66.7\%)

(50\%)

9

(50\%)

0.264

$$
>1.6
$$

Categorical variables were expressed as number (percentage), continuous variables were expressed as mean \pm SD \& median (range); •Mann Whitney U test; $\$$ Chi-square test; $p<0.05$ is significant. 
Table 3. Relation between clinicopathological features, flow cytometry markers and BCL-2 expression.

\begin{tabular}{|c|c|c|c|c|c|c|c|}
\hline \multirow{3}{*}{ Characteristics } & \multirow{2}{*}{\multicolumn{2}{|c|}{$\begin{array}{c}\text { All } \\
(\mathrm{N}=45)\end{array}$}} & \multicolumn{4}{|c|}{ BCL-2 } & \multirow{3}{*}{$p$-value } \\
\hline & & & \multicolumn{2}{|c|}{$<10 \%(\mathrm{~N}=8)$} & \multicolumn{2}{|c|}{$>10 \%(\mathrm{~N}=37)$} & \\
\hline & No. & $(\%)$ & No. & $(\%)$ & No. & $(\%)$ & \\
\hline \multicolumn{8}{|l|}{ Age (years) } \\
\hline Mean \pm SD & 56.31 & \pm 12.3 & 52.62 & \pm 12.83 & 57.10 & \pm 12.12 & \multirow{2}{*}{$0.298 \bullet$} \\
\hline Median (Range) & 54 & $(38-74)$ & 45.50 & $(42-73)$ & 54 & $(38-74)$ & \\
\hline$\leq 60$ years & 25 & $(55.6 \%)$ & 5 & $(20 \%)$ & 20 & $(80 \%)$ & \multirow{2}{*}{0.716} \\
\hline$>60$ years & 20 & $(44.4 \%)$ & 3 & $(15 \%)$ & 17 & $(85 \%)$ & \\
\hline \multicolumn{8}{|l|}{ Sex } \\
\hline Male & 26 & $(57.8 \%)$ & 6 & $(23.1 \%)$ & 20 & $(76.9 \%)$ & \multirow{2}{*}{0.435} \\
\hline Female & 19 & $(42.2 \%)$ & 2 & $(10.5 \%)$ & 17 & $(89.5 \%)$ & \\
\hline \multicolumn{8}{|l|}{ Rai classification } \\
\hline Stage $0,1 \& 2$ & 20 & $(44.4 \%)$ & 7 & $(35 \%)$ & 13 & $(65 \%)$ & \multirow{2}{*}{0.015} \\
\hline Stage $3 \& 4$ & 25 & $(55.6 \%)$ & 1 & $(4 \%)$ & 24 & $(96 \%)$ & \\
\hline \multicolumn{8}{|l|}{ Hepatomegally } \\
\hline Absent & 23 & $(51.1 \%)$ & 6 & $(26.1 \%)$ & 17 & $(73.9 \%)$ & \multirow{2}{*}{0.243} \\
\hline Present & 22 & $(48.9 \%)$ & 2 & $(9.1 \%)$ & 20 & $(90.9 \%)$ & \\
\hline \multicolumn{8}{|l|}{ Lymphadenopathy } \\
\hline Absent & 14 & $(31.1 \%)$ & 3 & $(21.4 \%)$ & 11 & $(78.6 \%)$ & \multirow{2}{*}{0.689} \\
\hline Present & 31 & $(68.9 \%)$ & 5 & $(16.1 \%)$ & 26 & $(83.9 \%)$ & \\
\hline \multicolumn{8}{|l|}{ Spleenomegally } \\
\hline Absent & 12 & $(26.7 \%)$ & 5 & $(41.7 \%)$ & 7 & $(58.3 \%)$ & \multirow{2}{*}{0.022} \\
\hline Present & 33 & $(73.3 \%)$ & 3 & $(9.1 \%)$ & 30 & $(90.9 \%)$ & \\
\hline \multicolumn{8}{|l|}{ WBCs $\left(\times 10^{3} / \mathrm{mm}^{3}\right)$} \\
\hline Mean \pm SD & 46.80 & \pm 31.71 & 25.85 & \pm 8.49 & 51.33 & \pm 33.11 & \multirow{2}{*}{$0.014 \bullet$} \\
\hline Median (Range) & 31.60 & $(17.50-122.80)$ & 25 & $(17.50-38)$ & 36 & $(17.50-122.80)$ & \\
\hline$<100 \times 10^{3} / \mathrm{mm}^{3}$ & 39 & $(86.7 \%)$ & 8 & $(20.5 \%)$ & 31 & $(79.5 \%)$ & \multirow{2}{*}{0.572} \\
\hline$\geq 100 \times 10^{3} / \mathrm{mm}^{3}$ & 6 & $(13.3 \%)$ & 0 & $(0 \%)$ & 6 & $(100 \%)$ & \\
\hline \multicolumn{8}{|c|}{ Absolute lymphocytes $\left(\times 10^{3} / \mathrm{mm}^{3}\right)$} \\
\hline Mean \pm SD & 26.27 & \pm 16.42 & 17.01 & \pm 6.67 & 28.28 & \pm 17.25 & \multirow{2}{*}{$0.085 \bullet$} \\
\hline Median (Range) & 18 & $(9.10-67)$ & 17 & $(9.10-26)$ & 19 & $(12.50-67)$ & \\
\hline$<30 \times 10^{3} / \mathrm{mm}^{3}$ & 33 & $(73.3 \%)$ & 8 & $(20.5 \%)$ & 25 & $(75.8 \%)$ & 00876 \\
\hline$\geq 30 \times 10^{3} / \mathrm{mm}^{3}$ & 12 & $(26.7 \%)$ & 0 & $(0 \%)$ & 12 & $(100 \%)$ & $0.08 / 8$ \\
\hline Platelet count $\left(\times 10^{3} / \mathrm{m}\right.$ & & & & & & & \\
\hline Mean \pm SD & 121.13 & \pm 47.20 & 125 & \pm 46.94 & 120.29 & \pm 47.86 & ( 778 \\
\hline Median (Range) & 111 & $(45-205)$ & 111 & $(76-200)$ & 112 & $(45-205)$ & 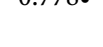 \\
\hline$<100 \times 10^{3} / \mathrm{mm}^{3}$ & 19 & $(42.2 \%)$ & 2 & $(10.5 \%)$ & 17 & $(89.5 \%)$ & 04256 \\
\hline$\geq 100 \times 10^{3} / \mathrm{mm}^{3}$ & 26 & $(57.8 \%)$ & 6 & $(23.1 \%)$ & 20 & $(76.9 \%)$ & 0.4559 \\
\hline Hemoglobin $(\mathrm{g} / \mathrm{dl})$ & & & & & & & \\
\hline Mean \pm SD & 10.73 & \pm 2.29 & 11.73 & \pm 2.73 & 10.51 & \pm 2.17 & 0191 \\
\hline Median (Range) & 10.20 & $(6.60-14.30)$ & 12.65 & $(7-14.30)$ & 10.10 & $(6.60-14)$ & 0.136 \\
\hline$<12 \mathrm{~g} / \mathrm{dl}$ & 25 & $(55.6 \%)$ & 2 & $(8 \%)$ & 23 & $(92 \%)$ & 0113 \\
\hline$\geq 12 \mathrm{~g} / \mathrm{dl}$ & 20 & (44.4\%) & 6 & $(30 \%)$ & 14 & $(70 \%)$ & 0.110 \\
\hline
\end{tabular}




\section{Continued}

$\begin{array}{cccccccc}\text { LDH (U/L) } & & & & & & & \\ \text { Mean } \pm \text { SD } & 354 & \pm 107.94 & 285 & \pm 36.25 & 368.91 & \pm 112.68 & 0.051 • \\ \text { Median (Range) } & 340 & (190-540) & 295 & (200-320) & 400 & (190-540) & \\ \leq 350 \mathrm{U} / \mathrm{L} & 23 & (51.1 \%) & 8 & (34.8 \%) & 15 & (65.2 \%) & 0.004 \\ >350 \mathrm{U} / \mathrm{L} & 22 & (48.9 \%) & 0 & (0 \%) & 22 & (100 \%) & \end{array}$

B2-microglobulin (mg/L)

$$
\begin{gathered}
\text { Mean } \pm \text { SD } \\
\text { Median (Range) } \\
<3.5 \mathrm{mg} / \mathrm{L} \\
\geq 3.5 \mathrm{mg} / \mathrm{L}
\end{gathered}
$$

Coomb's test

$$
\text { Negative }
$$$$
\text { Positive }
$$

Lymphocytic doubling time

$$
\begin{aligned}
& <12 \text { months } \\
& >12 \text { months }
\end{aligned}
$$

Cytogenetic analysis

Normal
del 13
del 11
Trisomy 12
del 17
Complex
CD38 (\%)
Mean \pm SD
Median (Range)
$<30 \%$
$>30 \%$
MCL-1 (\%)
Mean \pm SD
Median (Range)
$<25 \%$
$>25 \%$
ZAP-70
$<20 \%$
$>20 \%$

BCL-2/Bax ratio

$$
\begin{gathered}
\text { Mean } \pm \text { SD } \\
\text { Median (Range) } \\
\leq 1.6 \\
>1.6
\end{gathered}
$$

\subsection{1}

3

29

16

$\pm 0.99 \quad 2.03$

$$
(1.40-5)
$$

(35.6\%)

38

7

$\begin{array}{ll}(84.4 \%) & 8 \\ (15.6 \%) & 0\end{array}$

(21.1\%)

(0\%)

$\pm 0.44$

$(1.40-3)$

3.10

(27.6\%)

(0\%)

3

21

$$
\begin{gathered}
\pm 0.98 \\
(1.70-5) \\
(72.4 \%) \\
(100 \%)
\end{gathered}
$$

$0.006 \bullet$

0.037

$$
\text { (15.6\%) }
$$

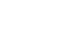

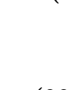

$\begin{array}{llllll}21 & (46.7 \%) & 7 & (33.3 \%) & 14 & (66.7 \%) \\ 24 & (53.3 \%) & 1 & (4.2 \%) & 23 & (95.8 \%)\end{array}$

(78.9\%)

(100\%)

0.321

$$
\text { (53.3\%) }
$$$$
\text { (4.2\%) }
$$$$
\text { (95.8\%) }
$$

0.017

$\begin{array}{ccccc}(44.4 \%) & 4 & (20 \%) & 16 & (80 \%) \\ (8.9 \%) & 2 & (50 \%) & 2 & (50 \%) \\ (11.1 \%) & 0 & (0 \%) & 5 & (100 \%) \\ (13.3 \%) & 2 & (33.3 \%) & 4 & (66.7 \%) \\ (11.1 \%) & 0 & (0 \%) & 5 & (100 \%) \\ (11.1 \%) & 0 & (0 \%) & 5 & (100 \%)\end{array}$

(80\%)

50\%)

(100\%)

66.7\%)

0.210

(100\%)

(100\%)

5

$\begin{array}{ccccccc}23.79 & \pm 25.41 & 3.53 & \pm 2.38 & 28.17 & \pm 26.03 & 0.013 \bullet \\ 8 & (0.30-91.59) & 4.41 & (0.30-5.95) & 27.61 & (0.60-91.59) & \\ 28 & (62.2 \%) & 8 & (28.6 \%) & 20 & (71.4 \%) & \\ 17 & (37.8 \%) & 0 & (0 \%) & 17 & (100 \%) & 0.017\end{array}$

\begin{tabular}{ccccccc}
60.60 & \pm 41.98 & 36.64 & \pm 36.07 & 65.78 & \pm 41.79 & $0.102 \bullet$ \\
91.30 & $(0.60-99.70)$ & 31.48 & $(1.10-99.70)$ & 97.47 & $(0.60-99.40)$ & \\
18 & $(40 \%)$ & 4 & $(22.2 \%)$ & 14 & $(77.8 \%)$ & 0.694 \\
27 & $(60 \%)$ & 4 & $(14.8 \%)$ & 23 & $(85.2 \%)$ & \\
& & & & & & \\
27 & $(60 \%)$ & 2 & $(7.4 \%)$ & 25 & $(92.6 \%)$ & 0.045 \\
18 & $(40 \%)$ & 6 & $(33.3 \%)$ & 12 & $(66.7 \%)$ & \\
2.01 & \pm 1.50 & 0.80 & \pm 0.26 & 2.27 & \pm 1.53 & \\
1.20 & $(0.30-5)$ & 0.85 & $(0.30-1.10)$ & 2 & $(0.30-5)$ & $0.006 \bullet$ \\
26 & $(57.8 \%)$ & 8 & $(30.8 \%)$ & 18 & $(69.2 \%)$ & \\
19 & $(42.2 \%)$ & 0 & $(0 \%)$ & 19 & $(100 \%)$ & 0.014 \\
\hline
\end{tabular}

Categorical variables were expressed as number (percentage), continuous variables were expressed as mean $\pm \mathrm{SD}$ \& median (range); $\bullet$ Mann Whitney $\mathrm{U}$ test; $\S$ Chi-square test; $p<0.05$ is significant. 
Table 4. BCL-2/Bax ratio as a predictor of overall response (OAR) of CLL patients to treatment; ROC curve analysis.

\begin{tabular}{cccccccc}
\hline Cut-off values & $\begin{array}{c}\mathrm{SN} \% \\
(95 \% \mathrm{CI})\end{array}$ & $\begin{array}{c}\mathrm{SP} \% \\
(95 \% \mathrm{CI})\end{array}$ & $\begin{array}{c}\text { PPV\% } \\
(95 \% \mathrm{CI})\end{array}$ & $\begin{array}{c}\text { NPV\% } \\
(95 \% \mathrm{CI})\end{array}$ & $\begin{array}{c}\text { Accuracy } \\
(95 \% \mathrm{CI})\end{array}$ & $\begin{array}{c}\text { AUROC } \\
(95 \% \mathrm{CI})\end{array}$ & $p$-value \\
\hline $\begin{array}{c}\text { BCL-2/Bax ratio } \\
\leq 1.6\end{array}$ & $\begin{array}{c}100 \% \\
(83.2-100)\end{array}$ & $\begin{array}{c}94.7 \% \\
(74-99.9)\end{array}$ & $\begin{array}{c}95.2 \% \\
(76.2-99.9)\end{array}$ & $\begin{array}{c}100 \% \\
(81.5-100)\end{array}$ & $\begin{array}{c}97.4 \% \\
(78.7-100)\end{array}$ & $\begin{array}{c}0.982 \\
(0.877-1.000)\end{array}$ & $<0.001$ \\
\hline
\end{tabular}

ROC curve: Receiver Operating Characteristic curve; SN: Sensitivity; SP: Specificity; PPV: Positive Predictive Value; NPV: Negative Predictive Value; AUROC: Area under Receiver Operating Characteristic curve; 95\% CI: 95\% Confidence Interval; $p<0.05$ is significant; Sig.: significance.

Table 5. Relation between clinicopathological features, flow cytometry markersand BCL-2/Bax ratio.

\begin{tabular}{|c|c|c|c|c|c|c|c|}
\hline \multirow{3}{*}{ Characteristics } & \multirow{2}{*}{\multicolumn{2}{|c|}{$\begin{array}{c}\text { All } \\
(\mathrm{N}=45)\end{array}$}} & \multicolumn{4}{|c|}{ BCL-2/Bax ratio } & \multirow{3}{*}{$p$-value } \\
\hline & & & \multicolumn{2}{|c|}{$\leq 1.6(\mathrm{~N}=26)$} & \multicolumn{2}{|c|}{$>1.6(\mathrm{~N}=19)$} & \\
\hline & No. & $(\%)$ & No. & (\%) & No. & $(\%)$ & \\
\hline \multicolumn{8}{|l|}{ Age (years) } \\
\hline Mean \pm SD & 56.31 & \pm 12.3 & 56.19 & \pm 12.92 & 56.47 & \pm 11.55 & \multirow{2}{*}{$0.927 \bullet$} \\
\hline Median (Range) & 54 & $(38-74)$ & 53.50 & $(38-74)$ & 54 & $(42-74)$ & \\
\hline$\leq 60$ years & 25 & $(55.6 \%)$ & 15 & $(60 \%)$ & 10 & $(55 \%)$ & \multirow{2}{*}{0.73} \\
\hline$>60$ years & 20 & $(44.4 \%)$ & 11 & $(55 \%)$ & 9 & $(45 \%)$ & \\
\hline \multicolumn{8}{|l|}{ Sex } \\
\hline Male & 26 & $(57.8 \%)$ & 16 & $(61.5 \%)$ & 10 & $(38.5 \%)$ & \multirow{2}{*}{0.550} \\
\hline Female & 19 & $(42.2 \%)$ & 10 & $(52.6 \%)$ & & $9(47.4 \%)$ & \\
\hline \multicolumn{8}{|l|}{ Rai classification } \\
\hline Stages $0,1 \& 2$ & 20 & $(44.4 \%)$ & 17 & $(85 \%)$ & 3 & $(15 \%)$ & \multirow{2}{*}{0.001} \\
\hline Stages $3 \& 4$ & 25 & $(55.6 \%)$ & 9 & $(36 \%)$ & 16 & $(64 \%)$ & \\
\hline \multicolumn{8}{|l|}{ Hepatomegally } \\
\hline Absent & 23 & $(51.1 \%)$ & 13 & $(56.5 \%)$ & 10 & $(43.5 \%)$ & \multirow{2}{*}{0.862} \\
\hline Present & 22 & $(48.9 \%)$ & 13 & $(59.1 \%)$ & 9 & $(40.9 \%)$ & \\
\hline \multicolumn{8}{|l|}{ Lymphadenopathy } \\
\hline Absent & 14 & $(31.1 \%)$ & 9 & $(64.3 \%)$ & 5 & $(35.7 \%)$ & \multirow{2}{*}{0.553} \\
\hline Present & 31 & $(68.9 \%)$ & 17 & $(54.8 \%)$ & 14 & $(45.2 \%)$ & \\
\hline \multicolumn{8}{|l|}{ Spleenomegally } \\
\hline Absent & 12 & $(26.7 \%)$ & 11 & $(91.7 \%)$ & 1 & $(8.3 \%)$ & \multirow{2}{*}{0.006} \\
\hline Present & 33 & $(73.3 \%)$ & 15 & $(45.5 \%)$ & 18 & $(54.5 \%)$ & \\
\hline \multicolumn{8}{|l|}{ WBCs $\left(\times 10^{3} / \mathrm{mm}^{3}\right)$} \\
\hline Mean \pm SD & 46.80 & \pm 31.71 & 47.90 & \pm 37.22 & 45.30 & \pm 23 & \multirow{2}{*}{$0.401 \bullet$} \\
\hline Median (Range) & 31.60 & $(17.50-122.80)$ & 33.25 & $(17.50-122.80)$ & 31.60 & $(17.50-112.50)$ & \\
\hline$<100 \times 10^{3} / \mathrm{mm}^{3}$ & 39 & $(86.7 \%)$ & 21 & $(53.8 \%)$ & 18 & $(46.2 \%)$ & \multirow{2}{*}{0.222} \\
\hline$\geq 100 \times 10^{3} / \mathrm{mm}^{3}$ & 6 & $(13.3 \%)$ & 5 & $(83.3 \%)$ & 1 & $(16.7 \%)$ & \\
\hline \multicolumn{8}{|c|}{ Absolute lymphocytes $\left(\times 10^{3} / \mathrm{mm}^{3}\right)$} \\
\hline Mean \pm SD & 26.27 & \pm 16.42 & 26.80 & \pm 17.42 & 25.55 & \pm 15.37 & \multirow{2}{*}{$0.881 \bullet$} \\
\hline Median (Range) & 18 & $(9.10-67)$ & 18.65 & $(9.10-67)$ & 17.30 & $(13-67)$ & \\
\hline$<30 \times 10^{3} / \mathrm{mm}^{3}$ & 33 & $(73.3 \%)$ & 19 & $(57.6 \%)$ & 14 & $(42.4 \%)$ & \multirow{2}{*}{0.964} \\
\hline$\geq 30 \times 10^{3} / \mathrm{mm}^{3}$ & 12 & $(26.7 \%)$ & 7 & $(58.3 \%)$ & 5 & $(41.7 \%)$ & \\
\hline \multicolumn{8}{|c|}{ Platelet count $\left(\times 10^{3} / \mathrm{mm}^{3}\right)$} \\
\hline Mean \pm SD & 121.13 & \pm 47.20 & 134.69 & \pm 48.89 & 102.57 & \pm 38.69 & 0025 \\
\hline Median (Range) & 111 & $(45-205)$ & 125.50 & $(45-205)$ & 90 & $(49-181)$ & 0.0336 \\
\hline$<100 \times 10^{3} / \mathrm{mm}^{3}$ & 19 & $(42.2 \%)$ & 7 & $(36.8 \%)$ & 12 & $(63.2 \%)$ & 0015 \\
\hline$\geq 100 \times 10^{3} / \mathrm{mm}^{3}$ & 26 & $(57.8 \%)$ & 19 & $(73.1 \%)$ & 7 & $(26.9 \%)$ & 0.013 \\
\hline
\end{tabular}




\section{Continued}

\begin{tabular}{|c|c|c|c|c|c|c|c|}
\hline \multicolumn{7}{|l|}{ Hemoglobin (g/dl) } & \multirow{3}{*}{$0.009 \bullet$} \\
\hline Mean \pm SD & 10.73 & \pm 2.29 & 11.56 & \pm 2.16 & 9.58 & \pm 2.01 & \\
\hline Median (Range) & 10.20 & $(6.60-14.30)$ & 12.50 & $(7-14.30)$ & 9.10 & $(6.60-14)$ & \\
\hline$<12 \mathrm{~g} / \mathrm{dl}$ & 25 & $(55.6 \%)$ & 9 & $(36 \%)$ & 16 & $(64 \%)$ & \multirow{2}{*}{0.001} \\
\hline$\geq 12 \mathrm{~g} / \mathrm{dl}$ & 20 & $(44.4 \%)$ & 17 & $(85 \%)$ & 3 & $(15 \%)$ & \\
\hline \multicolumn{8}{|l|}{$\mathrm{LDH}(\mathrm{U} / \mathrm{L})$} \\
\hline Mean \pm SD & 354 & \pm 107.94 & 305.76 & \pm 89.94 & 420 & \pm 96.37 & \multirow{2}{*}{$0.001 \bullet$} \\
\hline Median (Range) & 340 & $(190-540)$ & 300 & $(190-500)$ & 440 & $(210-540)$ & \\
\hline$\leq 350 \mathrm{U} / \mathrm{L}$ & 23 & $(51.1 \%)$ & 19 & $(82.6 \%)$ & 4 & $(17.4 \%)$ & \multirow{2}{*}{0.001} \\
\hline$>350 \mathrm{U} / \mathrm{L}$ & 22 & $(48.9 \%)$ & 7 & $(31.8 \%)$ & 15 & $(68.2 \%)$ & \\
\hline \multicolumn{8}{|c|}{ B2-microglobulin (mg/L) } \\
\hline Mean \pm SD & 2.91 & \pm 0.99 & 2.67 & \pm 0.97 & 3.25 & \pm 0.95 & \multirow{2}{*}{$0.046 \bullet$} \\
\hline Median (Range) & 3 & $(1.40-5)$ & 2 & $(1.40-4.40)$ & 3.30 & $(2-5)$ & \\
\hline$<3.5 \mathrm{mg} / \mathrm{L}$ & 29 & $(64.4 \%)$ & 18 & $(62.1 \%)$ & 11 & $(37.9 \%)$ & \multirow{2}{*}{0.433} \\
\hline$\geq 3.5 \mathrm{mg} / \mathrm{L}$ & 16 & $(35.6 \%)$ & 8 & $(50 \%)$ & 8 & $(50 \%)$ & \\
\hline \multicolumn{8}{|l|}{ Coomb's test } \\
\hline Negative & 38 & $(84.4 \%)$ & 24 & $(63.2 \%)$ & 14 & $(36.8 \%)$ & \multirow{2}{*}{0.114} \\
\hline Positive & 7 & $(15.6 \%)$ & 2 & $(28.6 \%)$ & 5 & $(71.4 \%)$ & \\
\hline \multicolumn{8}{|c|}{ Lymphocytic doubling time } \\
\hline$<12$ months & 21 & $(46.7 \%)$ & 18 & $(85.7 \%)$ & 3 & $(14.3 \%)$ & \multirow{2}{*}{$<0.001$} \\
\hline$>12$ months & 24 & $(53.3 \%)$ & 8 & $(33.3 \%)$ & 16 & $(66.7 \%)$ & \\
\hline \multicolumn{8}{|l|}{ Cytogenetic analysis } \\
\hline Normal & 20 & $(44.4 \%)$ & 15 & $(75 \%)$ & 5 & $(25 \%)$ & \multirow{6}{*}{0.001} \\
\hline del 13 & 4 & $(8.9 \%)$ & 3 & $(75 \%)$ & 1 & $(25 \%)$ & \\
\hline del 11 & 5 & $(11.1 \%)$ & 1 & $(20 \%)$ & 4 & $(80 \%)$ & \\
\hline Trisomy 12 & 6 & $(13.3 \%)$ & 6 & $(100 \%)$ & 0 & $(0 \%)$ & \\
\hline del 17 & 5 & $(11.1 \%)$ & 1 & $(20 \%)$ & 4 & $(80 \%)$ & \\
\hline Complex & 5 & $(11.1 \%)$ & 0 & $(0 \%)$ & 5 & $(100 \%)$ & \\
\hline \multicolumn{8}{|l|}{ CD38 (\%) } \\
\hline Mean \pm SD & 23.79 & \pm 25.41 & 11.76 & \pm 13.31 & 40.26 & \pm 28.94 & \multirow{2}{*}{$<0.001 \bullet$} \\
\hline Median (Range) & 8 & $(0.30-91.59)$ & 4.90 & $(0.30-42)$ & 38 & $(0.72-91.59)$ & \\
\hline$<30 \%$ & 28 & $(62.2 \%)$ & 23 & $(82.1 \%)$ & 5 & $(17.9 \%)$ & \multirow{2}{*}{$<0.001$} \\
\hline$>30 \%$ & 17 & $(37.8 \%)$ & 3 & $(17.6 \%)$ & 14 & $(82.4 \%)$ & \\
\hline \multicolumn{8}{|l|}{ MCL-1 (\%) } \\
\hline Mean \pm SD & 60.60 & \pm 41.98 & 49.14 & \pm 42.41 & 76.27 & \pm 36.89 & \multirow{2}{*}{$0.087 \bullet$} \\
\hline Median (Range) & 91.30 & $(0.60-99.70)$ & 38.03 & $(0.60-99.70)$ & 98 & $(9.70-99.20)$ & \\
\hline$<25 \%$ & 18 & $(40 \%)$ & 13 & $(72.2 \%)$ & 5 & $(27.8 \%)$ & \multirow{2}{*}{0.109} \\
\hline$>25 \%$ & 27 & $(60 \%)$ & 13 & $(48.1 \%)$ & 14 & $(51.9 \%)$ & \\
\hline \multicolumn{8}{|l|}{ BCL-2 (\%) } \\
\hline Mean \pm SD & 52.20 & \pm 29.24 & 46.47 & \pm 34.34 & 60.04 & \pm 18.45 & 0223 \\
\hline Median (Range) & 53.90 & $(2-93.20)$ & 48.20 & $(2-93.20)$ & 66.20 & $(35.70-93.20)$ & $0.223 \bullet$ \\
\hline$<10 \%$ & 8 & $(17.8 \%)$ & 8 & $(100 \%)$ & 0 & $(0 \%)$ & 0014 \\
\hline$>10 \%$ & 37 & $(82.2 \%)$ & 18 & $(48.6 \%)$ & 19 & (51.4\%) & 0.014 \\
\hline ZAB-70 & & & & & & & \\
\hline$<20 \%$ & 27 & $(60 \%)$ & 11 & $(40.7 \%)$ & 16 & $(59.3 \%)$ & 0005 \\
\hline$>20 \%$ & 18 & $(40 \%)$ & 15 & (83.3\%) & 3 & (16.7\%) & 0.005 \\
\hline
\end{tabular}

Categorical variables were expressed as number (percentage), continuous variables were expressed as mean \pm SD \& median (range); $\bullet$ Mann Whitney U test; $\S$ Chi-square test; $p<0.05$ is significant. 
Table 6. Relation between response and clinicopathological features, flow cytometry markers.

\begin{tabular}{|c|c|c|c|c|c|c|c|}
\hline \multirow{3}{*}{ Characteristics } & \multirow{2}{*}{\multicolumn{2}{|c|}{$\begin{array}{c}\text { All } \\
(\mathrm{N}=39)\end{array}$}} & \multicolumn{4}{|c|}{ Response } & \multirow{3}{*}{$p$-value } \\
\hline & & & \multicolumn{2}{|c|}{ No response $(\mathrm{N}=19)$} & \multicolumn{2}{|c|}{ OAR $(\mathrm{N}=20)$} & \\
\hline & No. & (\%) & No. & $(\%)$ & No. & (\%) & \\
\hline \multicolumn{8}{|l|}{ Age (years) } \\
\hline Mean \pm SD & 57.79 & \pm 12.01 & 56.57 & \pm 11.45 & 58.95 & \pm 12.71 & \multirow{2}{*}{$0.473 \bullet$} \\
\hline Median (Range) & 54 & $(39-74)$ & 54 & $(42-74)$ & 59 & $(39-74)$ & \\
\hline$\leq 60$ years & 20 & $(51.3 \%)$ & 10 & $(50 \%)$ & 10 & $(50 \%)$ & \multirow{2}{*}{$0.869 \$$} \\
\hline$>60$ years & 19 & $(48.7 \%)$ & 9 & $(47.4 \%)$ & 10 & $(52.6 \%)$ & \\
\hline \multicolumn{8}{|l|}{ Sex } \\
\hline Male & 23 & $(59 \%)$ & 10 & $(43.5 \%)$ & 13 & $(56.5 \%)$ & \multirow{2}{*}{$0.433 \S$} \\
\hline Female & 16 & $(41 \%)$ & 9 & $(56.3 \%)$ & 7 & $(43.8 \%)$ & \\
\hline \multicolumn{8}{|l|}{ Rai classification } \\
\hline Stages $0,1 \& 2$ & 16 & $(41 \%)$ & 3 & $(18.8 \%)$ & 13 & $(81.3 \%)$ & \multirow{2}{*}{$0.002 \S$} \\
\hline Stages $3 \& 4$ & 23 & $(59 \%)$ & 16 & $(69.6 \%)$ & 7 & $(30.4 \%)$ & \\
\hline \multicolumn{8}{|l|}{ Hepatomegally } \\
\hline Absent & 21 & $(53.8 \%)$ & 11 & $(52.4 \%)$ & 10 & $(47.6 \%)$ & \multirow{2}{*}{$0.621 \S$} \\
\hline Present & 18 & $(46.2 \%)$ & 8 & $(44.4 \%)$ & 10 & $(55.6 \%)$ & \\
\hline \multicolumn{8}{|l|}{ Lymphadenopathy } \\
\hline Absent & 13 & $(33.3 \%)$ & 5 & $(38.5 \%)$ & 8 & $(61.5 \%)$ & \multirow{2}{*}{$0.365 \S$} \\
\hline Present & 26 & $(66.6 \%)$ & 14 & $(53.8 \%)$ & 12 & $(46.2 \%)$ & \\
\hline \multicolumn{8}{|l|}{ Spleenomegally } \\
\hline Absent & 9 & $(23.1 \%)$ & 1 & $(11.1 \%)$ & 8 & $(88.9 \%)$ & \multirow{2}{*}{$0.020 \$$} \\
\hline Present & 30 & $(76.9 \%)$ & 18 & $(60 \%)$ & 12 & $(40 \%)$ & \\
\hline \multicolumn{8}{|l|}{ WBCs $\left(\times 10^{3} / \mathrm{mm}^{3}\right)$} \\
\hline Mean \pm SD & 49.44 & \pm 33.21 & 47.46 & \pm 23.50 & 51.32 & \pm 40.93 & \multirow{2}{*}{$0.407 \bullet$} \\
\hline Median (Range) & 36 & $(17.50-122.80)$ & 43 & $(17.50-112.50)$ & 32.35 & $(17.50-122.80)$ & \\
\hline$<100 \times 10^{3} / \mathrm{mm}^{3}$ & 33 & $(84.6 \%)$ & 18 & $(54.5 \%)$ & 15 & $(45.5 \%)$ & \multirow{2}{*}{$0.182 \S$} \\
\hline$\geq 100 \times 10^{3} / \mathrm{mm}^{3}$ & 6 & $(15.4 \%)$ & 1 & $(16.7 \%)$ & 5 & $(83.3 \%)$ & \\
\hline \multicolumn{8}{|l|}{$\begin{array}{c}\text { Absolute } \\
\text { lymphocytes } \\
\left(\times 10^{3} / \mathrm{mm}^{3}\right)\end{array}$} \\
\hline Mean \pm SD & 27.72 & \pm 17.09 & 26.61 & \pm 15.29 & 28.79 & \pm 18.97 & \multirow{2}{*}{$0.855 \bullet$} \\
\hline Median (Range) & 19.30 & $(9.10-67)$ & 19 & $(13-67)$ & 21.15 & $(9.10-67)$ & \\
\hline$<30 \times 10^{3} / \mathrm{mm}^{3}$ & 27 & $(69.2 \%)$ & 13 & $(48.1 \%)$ & 14 & $(51.9 \%)$ & \multirow{2}{*}{$0.915 \S$} \\
\hline$\geq 30 \times 10^{3} / \mathrm{mm}^{3}$ & 12 & $(30.8 \%)$ & 6 & $(50 \%)$ & 6 & $(50 \%)$ & \\
\hline \multicolumn{8}{|c|}{ Platelet count $\left(\times 10^{3} / \mathrm{mm}^{3}\right)$} \\
\hline Mean \pm SD & 121.61 & \pm 48.49 & 97.68 & \pm 33.79 & 144.35 & \pm 50.05 & \multirow{2}{*}{$0.004 \bullet$} \\
\hline Median (Range) & 112 & $(45-205)$ & 89 & $(49-180)$ & 159.50 & $(45-205)$ & \\
\hline
\end{tabular}




\section{Continued}

$\begin{array}{llllllll}<100 \times 10^{3} / \mathrm{mm}^{3} & 17 & (43.6 \%) & 13 & (76.5 \%) & 4 & (23.5 \%) & 0.002 \S \\ \geq 100 \times 10^{3} / \mathrm{mm}^{3} & 22 & (56.4 \%) & 6 & (27.3 \%) & 16 & (72.7 \%) & \end{array}$

Hemoglobin (g/dl)

Mean \pm SD
Median (Range)

$<11 \mathrm{~g} / \mathrm{dl}$

$\geq 11 \mathrm{~g} / \mathrm{dl}$

LDH (U/L)

Mean \pm SD

Median (Range)

$\leq 350 \mathrm{U} / \mathrm{L}$

$>350 \mathrm{U} / \mathrm{L}$

B2-microglobulin (mg/L)

Mean \pm SD
Median (Range)
$<3.5 \mathrm{mg} / \mathrm{L}$
$\geq 3.5 \mathrm{mg} / \mathrm{L}$

Coomb's test

Negative
Positive

Lymphocytic doubling time

$$
\begin{aligned}
& <12 \text { months } \\
& >12 \text { months }
\end{aligned}
$$

Cytogenetic analysis

$$
\text { Normal }
$$

del 11

Trisomy 12

del 17

Complex

CD38 (\%)

$$
\text { Mean } \pm \text { SD }
$$

Median (Range)

$$
<30 \%
$$

$>30 \%$

$$
\begin{gathered}
10.79 \\
10.20 \\
22 \\
17
\end{gathered}
$$

10.20

$\pm 2.25$

(6.60 - 14.30)

(56.4\%)

(43.6\%)

363.84

$\pm 108.13$

(190 - 540)

(46.2\%)

(53.8\%)

21

(53.8\%)

3.01

3

24

15

$$
\pm 0.97
$$

(1.80 - 5)

(61.5\%)

(38.5\%)

(82.1\%)

(17.9\%)

7

18

21

(46.2\%)

(53.8\%)

3

$\begin{array}{ll}(43.6 \%) & 5 \\ (7.7 \%) & 1 \\ (10.3 \%) & 3 \\ (12.8 \%) & 0 \\ (12.8 \%) & 5 \\ (12.8 \%) & 5\end{array}$

25.94

$$
\pm 26.21
$$

22

23

16

(0.30 - 91.59)

(59\%)

(41\%)

24

(61.5\%)

15

9.59
9.10
16
3

419.47

4

$\pm 2.01$

(6.60 - 14)

$$
\text { (72.7\%) }
$$

(17.6\%)

$$
\pm 96.29
$$

(210 - 540)

(22.2\%)

(71.4\%)

11.9
12.95
6
14

11.93

$\pm 1.87$

(7.70 - 14.30)

0.002 •

$6 \quad(27.3 \%)$

$14 \quad(82.4 \%)$

$0.001 \S$

(7)

\pm 0.95
$(2-5)$
$(45.8 \%)$

311

$+96.56$

(190 - 500)

0.002 •

$\begin{array}{lll}14 & (77.8 \%) & \\ 6 & (28.6 \%) & 0.002 \S\end{array}$




\begin{tabular}{|c|c|c|c|c|c|c|c|}
\hline MCL-1 (\%) & & & & & & & \\
\hline Mean \pm SD & 64.98 & \pm 41.21 & 80.16 & \pm 34.13 & 50.55 & \pm 42.94 & \multirow{2}{*}{$0.177 \bullet$} \\
\hline Median (Range) & 96.60 & $(0.60-99.70)$ & 98 & $(9.70-99.20)$ & 38.03 & $(0.60-99.70)$ & \\
\hline$<25 \%$ & 14 & $(35.9 \%)$ & 4 & $(28.6 \%)$ & 10 & $(71.4 \%)$ & \multirow{2}{*}{$0.060 \$$} \\
\hline$>25 \%$ & 25 & $(64.1 \%)$ & 15 & $(60 \%)$ & 10 & $(40 \%)$ & \\
\hline \multicolumn{8}{|l|}{ BCL-2 (\%) } \\
\hline Mean \pm SD & 56.72 & \pm 27.64 & 59.74 & \pm 18.60 & 53.86 & \pm 34.39 & \multirow{2}{*}{$0.855 \bullet$} \\
\hline Median (Range) & 66.10 & $(2-93.20)$ & 66.20 & $(35.70-93.20)$ & 61.45 & $(2-93.20)$ & \\
\hline$<10 \%$ & 5 & $(12.8 \%)$ & 0 & $(0 \%)$ & 5 & $(100 \%)$ & \multirow{2}{*}{$0.047 \S$} \\
\hline$>10 \%$ & 34 & $(87.2 \%)$ & 19 & $(55.9 \%)$ & 15 & $(44.1 \%)$ & \\
\hline \multicolumn{8}{|l|}{ BCL-2/Bax ratio } \\
\hline Mean \pm SD & 2.16 & \pm 1.54 & 3.50 & \pm 1.10 & 0.89 & \pm 0.32 & \multirow{2}{*}{$<0.001 \bullet$} \\
\hline Median (Range) & 1.50 & $(0.30-5)$ & 3.70 & $(1-5)$ & 0.95 & $(0.30-1.60)$ & \\
\hline$\leq 1.6$ & 21 & $(53.8 \%)$ & 1 & $(4.8 \%)$ & 20 & $(95.2 \%)$ & \multirow{2}{*}{$<0.001$ \$ } \\
\hline$>1.6$ & 18 & $(46.2 \%)$ & 18 & $(100 \%)$ & 0 & (0\%) & \\
\hline
\end{tabular}

Categorical variables were expressed as number (percentage), continuous variables were expressed as mean \pm SD \& median (range); •Mann Whitney $\mathrm{U}$ test; $\S$ Chi-square test; $p<0.05$ is significant.

Although MCL-1 expression was not significantly affect the treatment response $(p=0.060)$, better overall response was associated with low MCL-1 expression, as 10/14 of those with low MCL-1developed objective response to treatment $(\mathrm{CR}+\mathrm{PR})$.

Low BCL-2 expression was significantly associated with achieving treatment response but when $\mathrm{CR}$ and $\mathrm{PR}$ were calculated together $(p=0.047)$, as all patients with low expression of BCL-2 were achieving overall response, and the 19 non responding patients were having high $\mathrm{BCL}-2$ expression. Also low BCL2/Bax ratio was significantly associated with better treatment response ( $\mathrm{CR}+\mathrm{PR})$ ( $p=0.001$ ); among the 39 patients who started treatment at diagnosis, responding patients had statistically significant lower BCL-2/Bax ratio mean than nonresponding patients $(0.89 \pm 0.32$ vs. $3.5 \pm 1.1$, respectively; $p<0.001)$, and none of the patients in the group with high BCL-2/Bax ratio achieving any response, while $95 \%$ of responding patients had low BCL-2/Bax ratio.

\subsection{Time to Start Treatment}

Our patients were grouped according to Rai staging system, to two categories; low risk group (stage 0 -II) and high risk (stage III-IV). The low risk group (20 patients) was followed up to detect time to start treatment and to determine factors that influence that time, so 20 patients were followed for up to 18 months; 6 of them didn't need to start treatment. The time to start chemotherapy treatment ranging from 2 to 14 months, we found a significant difference between patients $\leq 60$ years and those $>60$ years as regard time to start treatment ( $p=$ 0.026). Patients with high BCL-2 expression and those with high BCL-2/Bax ratio experienced shorter time to start treatment, but that was not statistically significant $(p>0.05)$ Table 7, Figure 3 and Figure 4. 
Table 7. Relation between time to start treatment in low risk group CLL patients and prognostic factors.

\begin{tabular}{|c|c|c|c|c|c|c|c|}
\hline \multirow[t]{2}{*}{ Characteristics } & \multicolumn{2}{|c|}{ All $(\mathrm{N}=20)$} & \multicolumn{4}{|c|}{ Time to start treatment (months) } & \multirow{2}{*}{$p$-value } \\
\hline & No. & $(\%)$ & Mean & $\pm \mathrm{SD}$ & Median & (Range) & \\
\hline \multicolumn{8}{|l|}{ Age } \\
\hline$\leq 60$ years & 12 & $(60 \%)$ & 11.16 & \pm 3.37 & 12.50 & $(4-14)$ & \multirow{2}{*}{$0.026 \bullet$} \\
\hline$>60$ years & 8 & $(40 \%)$ & 7.75 & \pm 3.28 & 7.50 & $(2-12)$ & \\
\hline \multicolumn{8}{|l|}{ Sex } \\
\hline Male & 14 & $(70 \%)$ & 9.85 & \pm 4.14 & 11.5 & $(2-14)$ & \multirow{2}{*}{$0.919^{*}$} \\
\hline Female & 6 & $(30 \%)$ & 9.66 & \pm 2.58 & 9 & $(7-14)$ & \\
\hline \multicolumn{8}{|l|}{ Hepatomegally } \\
\hline Absent & 11 & $(55 \%)$ & 9.63 & \pm 3.58 & 11 & $(4-14)$ & \multirow{2}{*}{$0.833^{\star}$} \\
\hline Present & 9 & $(45 \%)$ & 10 & \pm 4 & 10 & $(2-14)$ & \\
\hline \multicolumn{8}{|l|}{ Lymphadenopathy } \\
\hline Absent & 7 & $(35 \%)$ & 10.14 & \pm 4.18 & 11 & $(2-14)$ & \multirow{2}{*}{$0.769^{\star}$} \\
\hline Present & 13 & $(65 \%)$ & 9.61 & \pm 3.54 & 8 & $(4-14)$ & \\
\hline \multicolumn{8}{|l|}{ Spleenomegally } \\
\hline Absent & 11 & $(55 \%)$ & 10.45 & \pm 3.38 & 12 & $(4-14)$ & \multirow{2}{*}{$0.394^{*}$} \\
\hline Present & 9 & $(45 \%)$ & 9 & \pm 4.06 & 10 & $(2-14)$ & \\
\hline \multicolumn{8}{|l|}{ WBCs } \\
\hline$<100 \times 10^{3} / \mathrm{mm}^{3}$ & 15 & $(75 \%)$ & 10.46 & \pm 3.52 & 11 & $(4-14)$ & \multirow{2}{*}{$0.157^{\circ}$} \\
\hline$\geq 100 \times 10^{3} / \mathrm{mm}^{3}$ & 5 & $(25 \%)$ & 7.80 & \pm 3.76 & 8 & $(2-12)$ & \\
\hline \multicolumn{8}{|c|}{ Absolute lymphocytes } \\
\hline$<30 \times 10^{3} / \mathrm{mm}^{3}$ & 13 & $(65 \%)$ & 11.15 & \pm 3.23 & 12 & $(4-14)$ & \multirow{2}{*}{$0.018 \bullet$} \\
\hline$\geq 30 \times 10^{3} / \mathrm{mm}^{3}$ & 7 & $(35 \%)$ & 7.28 & \pm 3.25 & 7 & $(2-12)$ & \\
\hline \multicolumn{8}{|c|}{ Lymphocytic doubling time } \\
\hline$<12$ months & 14 & $(70 \%)$ & 10.14 & \pm 3.63 & 10.50 & $(2-14)$ & \multirow{2}{*}{$0.539^{\star}$} \\
\hline$>12$ months & 6 & $(30 \%)$ & 9 & \pm 4 & 9.50 & $(4-14)$ & \\
\hline \multicolumn{8}{|l|}{ Cytogenetic analysis } \\
\hline Normal & 12 & $(60 \%)$ & 9.58 & \pm 3.50 & 9.50 & $(4-14)$ & \multirow{4}{*}{$0.946 \ddagger$} \\
\hline del 13 & 3 & $(15 \%)$ & 10 & \pm 3.46 & 8 & $(8-14)$ & \\
\hline del 11 & 1 & $(5 \%)$ & 11 & & & & \\
\hline Trisomy 12 & 4 & $(20 \%)$ & 10 & \pm 5.65 & 12 & $(2-14)$ & \\
\hline CD38 & & & & & & & \\
\hline$<30 \%$ & 17 & $(85 \%)$ & 10.17 & \pm 3.35 & 11 & $(2-14)$ & 0 200* \\
\hline$>30 \%$ & 3 & $(15 \%)$ & 7.66 & \pm 5.50 & 5 & $(4-14)$ & 0.200 \\
\hline MCL-1 & & & & & & & \\
\hline$<25 \%$ & 9 & $(45 \%)$ & 10.66 & \pm 4.03 & 12 & $(2-14)$ & 0232 \\
\hline$>25 \%$ & 11 & $(55 \%)$ & 9.09 & \pm 3.38 & 8 & $(4-14)$ & 0.2006 \\
\hline BCL-2 & & & & & & & \\
\hline$<10 \%$ & 7 & $(35 \%)$ & 11.71 & \pm 2.75 & 13 & $(8-14)$ & 0077 \\
\hline$>10 \%$ & 13 & $(65 \%)$ & 8.76 & \pm 3.78 & 8 & $(2-14)$ & 0.076 \\
\hline ZAB-70 & & & & & & & \\
\hline$<20 \%$ & 6 & $(30 \%)$ & 8.50 & \pm 3.88 & 8 & $(4-14)$ & $0214 *$ \\
\hline$>20 \%$ & 14 & $(70 \%)$ & 10.35 & \pm 3.58 & 11 & $(2-14)$ & 0.314 \\
\hline BCL-2/Bax ratio & & & & & & & \\
\hline$\leq 1.6$ & 17 & $(85 \%)$ & 10.35 & \pm 3.69 & 11 & $(2-14)$ & 0077 \\
\hline$>1.6$ & 3 & $(15 \%)$ & 6.66 & \pm 1.52 & 7 & $(5-8)$ & $0.07 / 6$ \\
\hline
\end{tabular}

Categorical variables were expressed as number (percentage), continuous variables were expressed as mean \pm SD \& median (range); ${ }^{*}$ Independent samples Student's t-test; •Mann Whitney U test; ${ }^{*}$ Kraskall Wallis $\mathrm{H}$ test; $p<0.05$ is significant. 


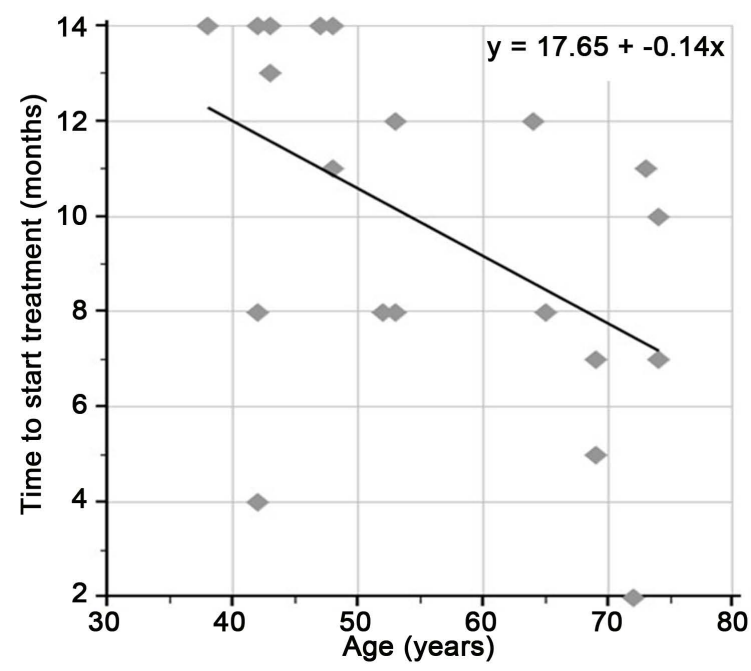

(a)

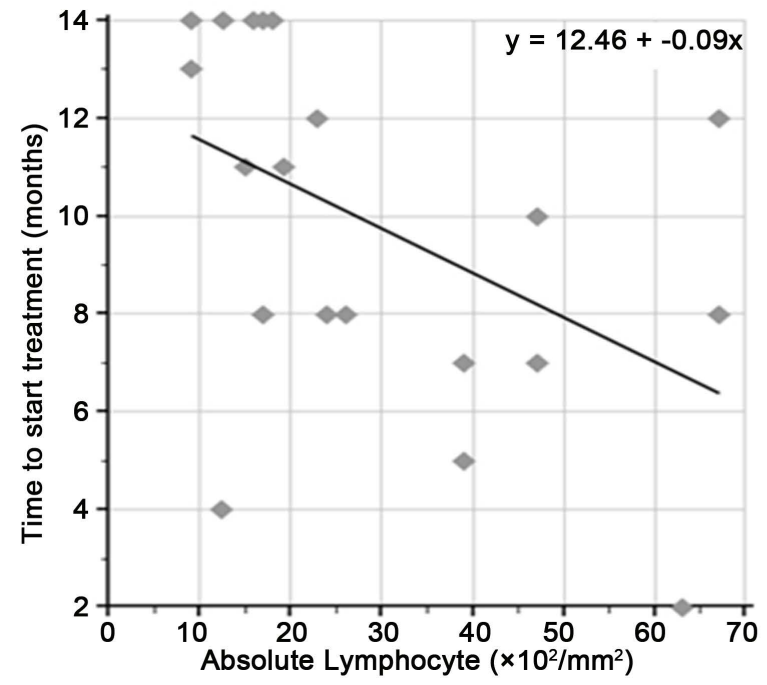

(c)

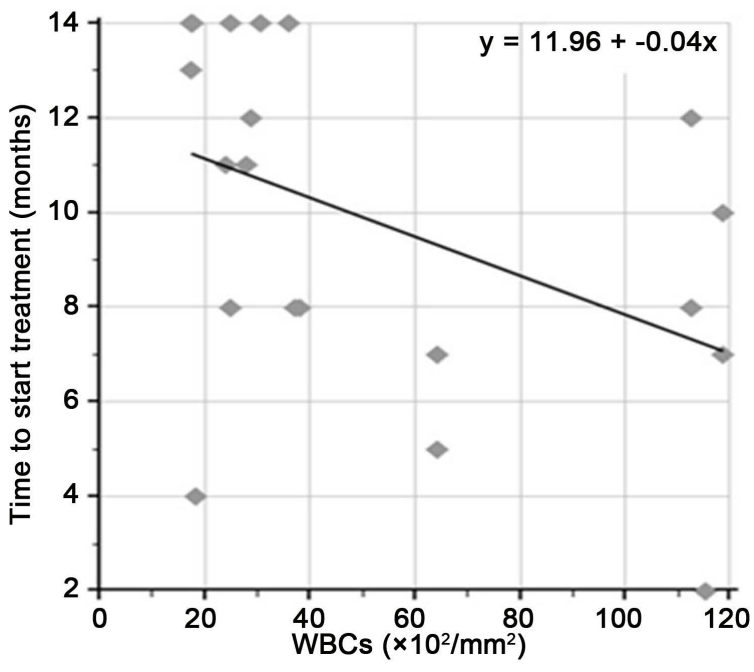

(b)

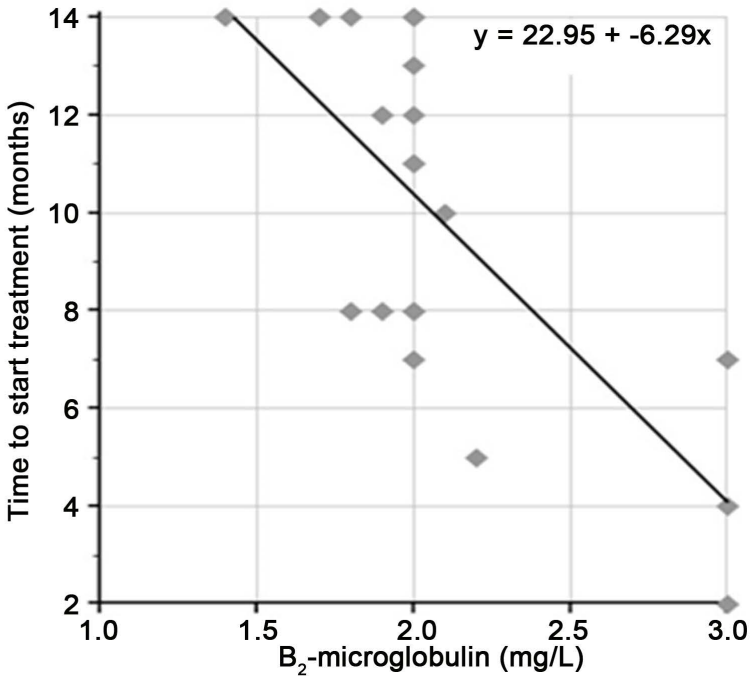

(d)

Figure 3. Scatter plot with regression line shows indirect correlation between (a) Age (years); (b) WBCs $\left(\times 10^{3} / \mathrm{mm}^{3}\right)$; (c) Absolute lymphocyte count $\left(\times 10^{3} / \mathrm{mm}^{3}\right)$; (d) B2-microglobulin (mg/L) and time to start first treatment (months).

Also we found a significant indirect correlation between age, WBCS count, Absolute lymphocyte count, B2-microglobulin and time to start first treatment (months) Table 8.

\section{Discussion}

Nowadays with the development of novel treatment options it necessitate the identification of patients, with unfavorable prognostic features, who are more liable for early progression and who would gain the most benefit from early interference with targeted treatment. For this reason, identification of prognostic factors of CLL is the interest of all researchers [18]. As known BCL-2 is working as an anti-apoptosis and it is an integral inner mitochondrial membrane protein; its over-expression prevent the apoptotic death of a pro-B-lymphocyte cell line. Thus, BCL-2 is unique among proto-oncogene, being localized in mitochondria 


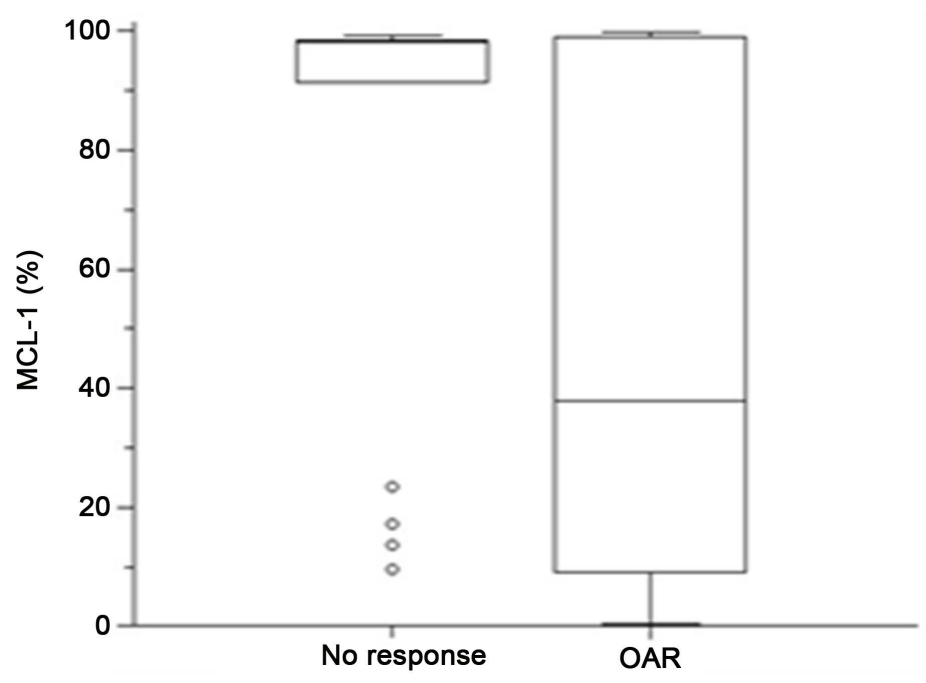

(a)

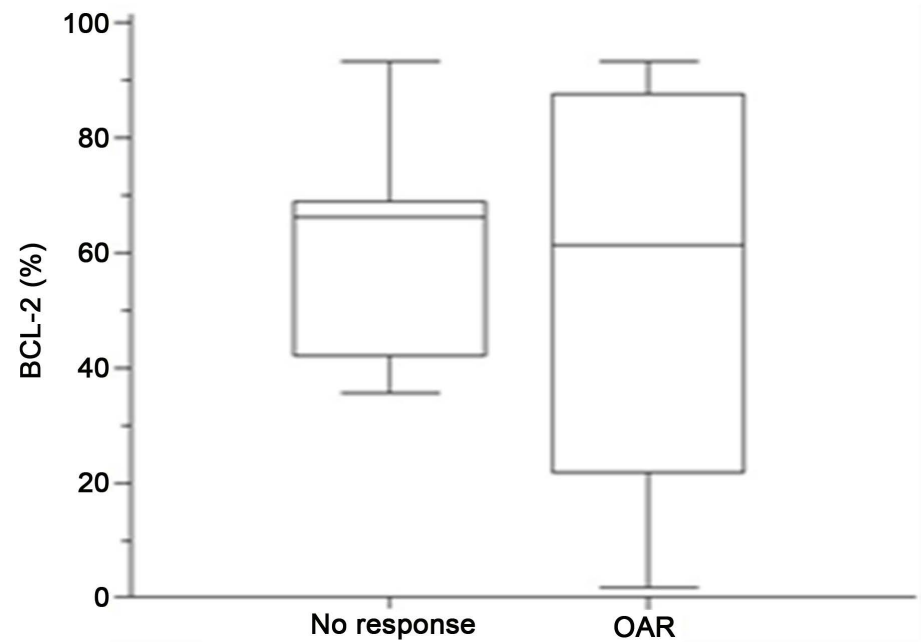

(b)

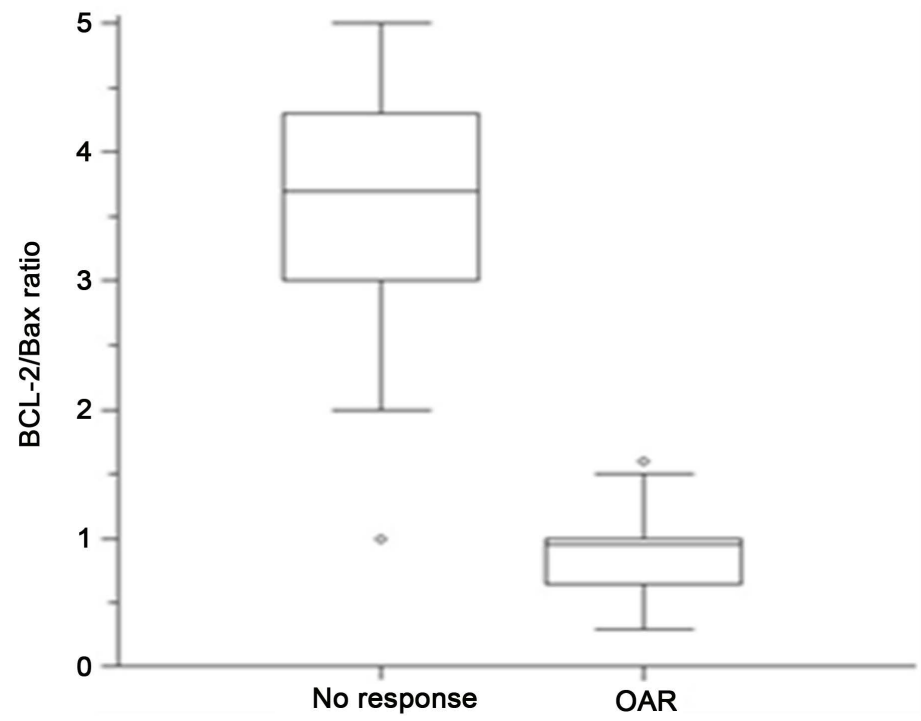

(c)

Figure 4. Box plot shows comparison between non-responder and responder as regard (a) MCL-1 (\%); (b) BCL-2 (\%) and (c) BCL-2/Bax ratio. 
Table 8. Correlation between time to start treatment (months) and prognostic markers.

\begin{tabular}{ccc}
\hline Variables & \multicolumn{2}{c}{ Time to start first treatment (months) } \\
\cline { 2 - 3 } & $\mathrm{r}$ & $p$-value \\
\hline Age $($ years $)$ & -0.498 & 0.025 \\
WBCs $\left(\times 10^{3} / \mathrm{mm}^{3}\right)$ & -0.511 & 0.021 \\
Absolute lymphocytes $\left(\times 10^{3} / \mathrm{mm}^{3}\right)$ & -0.507 & 0.023 \\
Platelet count $\left(\times 10^{3} / \mathrm{mm}^{3}\right)$ & -0.274 & 0.242 \\
Hemoglobin $(\mathrm{g} / \mathrm{dl})$ & -0.203 & 0.392 \\
LDH (U/L) & -0.010 & 0.967 \\
B2-microglobulin $(\mathrm{mg} / \mathrm{L})$ & -0.665 & 0.001 \\
CD38 (\%) & -0.209 & 0.376 \\
MCL-1 (\%) & -0.239 & 0.310 \\
BCL-2 (\%) & -0.305 & 0.191 \\
BCL-2/Bax ratio & -0.388 & 0.091 \\
\hline
\end{tabular}

r: Spearman's rank correlation coefficient; $p<0.05$ is significant.

and interfering with apoptosis independent of cell division promotion [19]. Although the exact mechanism of BCL-2 action is not known, high expression of BCL-2 correlates with increased cell survival through reduction of apoptosis by interfering sequences that lead to apoptosis [20], We have known several members of anti-apoptotic and pro-apoptotic BCL-2 family, but MCL-1 is the most significant anti-apoptotic protein associated with normal as well as malignant $\mathrm{B}$ lymphocytes, it is essential during both lymphoid development and maintenance of mature $\mathrm{T}$ and $\mathrm{B}$ lymphocytes [21].

Our study showed that 27 patients (60\%) were positive MCL-1, while Anurag Saxena et al. [15] found that $72 \%$ of patients were positive MCL-1. No significance between MCL-1 and Rai classification was found, and this in accordance with Anurag Saxena et al. [15], who reported that MCL-1 did not has significant association with Rai stage. This in contrary to Pepper et al. [6], who reported that, MCL-1 expression was significantly correlated with stage of disease $(p<0.001)$, and this is may be explained partially by larger sample size (185 patients). MCL-1 showed positive significant correlation with expression of CD38 in our study $(p=0.002)$, and this in agreement with Pepper et al. [6] $(p<0.001)$.

In our study an association between low MCL-1 levels and ability to gain overall response (10/14) to treatment was detected, in Anurag Saxena study [15], and in Kitada's report [22], all patients with CR had low MCL-1 levels. Also Kitada's reported that MCL-1 is the only anti-apoptotic protein which was identified to be associated with in vitro resistance to chlormabucil and fludarabine and significantly lower CR rates in patients with CLL [22]. MCL-1upregulation in CLL patients may be related to certain growth factors e.g., interleukin-4 (IL-4), and IL-13 [23], or alternation in structure of MCL-1 gene [24], that lead to MCL-1 protein persistent elevations in those patients. Marschitz et al. [25], mentioned that BCL-2 strong expression is a constant feature of CLL cells, Srinivas et al. [16] pointed that the cut-off level was defined as more than $10 \%$, In our study, 37 cases had BCL-2+ cells $>10 \%$ and this represented $82.2 \%$ of the studied cases , Shinichi et al. [26], reported that the levels of BCL-2 protein expres- 
sion were $60 \%$ of B-CLL patients, While in study by Lazaridou et al. [27], the levels of BCL-2 protein expression were $76.3 \%$ of the CLL patients, and Mendez et al. [28], showed that the levels of BCL-2 protein expression were $77 \%$ of B-CLLs. This difference may be due to late diagnosis, or high Rai classification in our patients. Marschitz et al. [25], found higher levels of BCL-2 in patients with progressive disease. In our study, we found positive significant correlation between Rai staging and splenomegaly with BCL-2 expression levels $(p<0.005)$. Marschitz et al. [25]), and Anurag et al. [15] reported that BCL-2 expression levels were not correlated with Rai stages of disease this is, possibly due to different methods of measurement (e.g. western blot technique). In our study, high BCL-2 expression was correlated with short lymphocytic doubling time, high LDH, high serum $\beta 2 \mathrm{M}$, high CD38 expression and low ZAP-70 expression. As regard the treatment response, we found low BCL-2 expression was significantly associated with better treatment response only when CR and PR were considered together $(p=0.047)$, and this in agreement with Schimmer et al. [29], who pointed that, aberrant expression of BCL-2 was associated with poor response to chemotherapy and decreased overall survival.

Regard the clinical impact of BCL-2/Bax ratio in the present study; as independent prognostic factor in CLL patients, we detected that a higher BCL-2/Bax ratio strongly correlated with some unfavorable clinical presentations like low $\mathrm{HB}$, low platelets, high $\mathrm{LDH}$ and splenomegaly, also significant associations detected between high BCL-2/Bax ratio and indicators of higher tumor burden (B2M, and LDT, Rai stages) and other prognostic markers such CD38, and cytogenetics, and CD38 overexpression is well known to has adverse prognostic effect in CLL [30] [31], and there were significant correlations between BCL-2, MCL-1, and BCL-2/Bax ratio with CD38 expression, so estimation of BCL-2, MCL-1 and BCL-2/Bax ratio expressions in CLL cases could be used as predictors of bad prognosis. Kitada et al. [22] mentioned that in his result increased BCL-2/Bax ratio was associated with high total leucocyte count, also Anurag Saxena et al. [15], noted that in BCL-2/Bax positive patients the LDT was significantly longer, which suggests a significant correlation between this ratio and high proliferation. In our study, low BCL-2/Bax ratio showed a statistically significant association with treatment response when $\mathrm{CR}+\mathrm{PR}$ were calculated together, and this in agreement with Anurag Saxena et al. [15], who noted BCL-2/ Bax ratio was significantly associated with treatment response $(0.89 \pm 0.53[\mathrm{CR}+$ $\mathrm{PR}$ ] vs. $3.38 \pm 4.47$ [NR] $(p=0.011))$ with others results it there was an association between a high BCL-2/Bax ratio and treatment resistance [11] [12] [13]. Correlations between BCL-2/Bax ratios and chlorambucil was demonstrated in some in vitro studies [32], with fludarabine-induced apoptosis [33] [34], and also with steroid-induced apoptosis [35]. In CLL patients high BCL-2/Bax ratio may reflect resistant clones [32], however, this point still with some controversy in literature, as some investigators have not found any correlation between in vitro or in vivo sensitivity to chlorambucil, fludarabine, and BCL-2, BAX, and their ratio [18] [22]. Also, Zaja et al., failed in his study to show any correlation 
between BCL-2 and treatment response [36]. These controversies may be explained by variability in the comparing and measuring protein levels by using different standard, also the samples collection time in relation to therapy timing may play role in these variabilities.

\section{Conclusion}

By using a flow cytometric method, we detected the prognostic power of MCL-1, BCL-2, and BCL-2/Bax ratio, which is an easy method used in routine laboratory practice. Also we defined the correlations of these proteins expressions with chemo-resistance and clinical outcome in CLL patients. So MCL-1, BCL-2, and BCL-2/Bax ratio can be used to determine CLL cases that can be targeted by new BCL-2 inhibitors therapy.

\section{Conflict of Interest}

The authors have declared no conflict of interest.

\section{References}

[1] Rozman, C. and Montserrat, E. (1995) Chronic Lymphocytic Leukemia. The New England Journal of Medicine, 333, 1052-1057. https://doi.org/10.1056/NEJM199510193331606

[2] Chiorazzi, N., Rai, K.R. and Ferrarini, M. (2005) Chronic Lymphocytic Leukemia. The New England Journal of Medicine, 352, 804-815. https://doi.org/10.1056/NEJMra041720

[3] Krober, A., Seiler, T., Benner, A., Bullinger, L., Bruckle, E., Lichter, P., et al. (2002) $\mathrm{V}(\mathrm{H})$ Mutation Status, CD38 Expression Level, Genomic Aberrations, and Survival in Chronic Lymphocytic Leukemia. Blood, 100, 1410-1416.

[4] Kipps, T.J. (1997) Chronic Lymphocytic Leukemia. Current Opinion in Hematology, 4, 268-276. https://doi.org/10.1097/00062752-199704040-00008

[5] Caligaris-Cappio, F. (1996) B-Chronic Lymphocytic Leukemia: A Malignancy of Anti-Self B Cells. Blood, 87, 2615-2620.

[6] Pepper, C., Hooper, K., Thomas, A., et al. (2001) BCL-2 Antisense Oligonucleotides Enhance the Cytotoxicity of Chlorambucil-Induced Apoptosis in Chronic Lymphocytic Leukemia. Leukemia \& Lymphoma, 42, 491-498. https://doi.org/10.3109/10428190109064606

[7] Scarfò, L. and Ghia, P. (2013) Reprogramming Cell Death: BCL2 Family Inhibition in Hematological Malignancies. Immunology Letters, 155, 36-39. https://doi.org/10.1016/j.imlet.2013.09.015

[8] Cimmino, A., Calin, G.A., Fabbri, M., et al. (2005) miR-15 and miR-16 Induce Apoptosis by Targeting BCL2. Proceedings of the National Academy of Sciences of the United States of America, 102, 13944-13949. https://doi.org/10.1073/pnas.0506654102

[9] Pepper, C., Hoy, T. and Bentley, P. (1998) Elevated BCL-2/Bax Are a Consistent Feature of Apoptosis Resistance in B-Cell Chronic Lymphocytic Leukemia and Are Correlated with in Vivo Chemoresistance. Leukemia \& Lymphoma, 28, 355-361. https://doi.org/10.3109/10428199809092690

[10] Korsmeyer, S.J., Shutter, J.R., Veis, D.J., Merry, D.E. and Oltvai, Z.N. (1993) BCL-2/ Bax: A Rheostat That Regulates an Anti-Oxidant Pathway and Cell Death. Seminars 
in Cancer Biology, 4, 327-332.

[11] Pepper, C., Bentley, P. and Hoy, T. (1996) Regulation of Clinical Chemoresistance by BCL-2 and Bax Oncoproteins in B-Cell Chronic Lymphocytic Leukemia. British Journal of Haematology, 95, 513-517. https://doi.org/10.1046/j.1365-2141.1996.d01-1927.x

[12] Thomas, A., El Rouby, S., Reed, J.C., Krajewski, S., Silber, R., Potmesil, M., et al. (1996) Drug Induced Apoptosis in B-Cell Chronic Lymphocytic Leukemia: Relationship between p53 Gene Mutation and BCL-2/Bax Proteins in Drug Resistance. Oncogene, 12, 1055-1062.

[13] Pepper, C., Hoy, T. and Bentley, D.P. (1997) BCL-2/Bax Ratios in Chronic Lymphocytic Leukaemia and Their Correlation with in Vitro Apoptosis and Clinical Resistance. British Journal of Cancer, 76, 935-938. https://doi.org/10.1038/bjc.1997.487

[14] Klobusicka, M., Kusenda, J. and Babusikova, O. (2002) Immunocytochemical Detection of BCL-2 and p53 Proteins in B-Chronic Lymphocytic Leukemia Patients. Neoplasma, 49, 387-397.

[15] Anurage Saxena, A., Viswanathan, S., Moshynska, O., et al. (2004) MCL-1 and BCL2/Bax Ratio Are Associated with Treatment Response but Not with Rai Stage in BCell Chronic Lymphocytic Leukemia. American Journal of Hematology, 75, 22-33. https://doi.org/10.1002/ajh.10453

[16] Srinivas, G., Kusumary, P., Krishnav, M., et al. (2000) Mutant p53 Protein, BCL-2/ Bax Ratio and Apoptosis in Pediatric Acute Lymphoblastic Leukemia. Journal of Cancer Research and Clinical Oncology, 126, 62-67. https://doi.org/10.1007/s004320050010

[17] Cheson, B.D., Bennett, J.M., Grever, M., Kay, N., Keating, M.J., O’Brien, S., et al. (1996) National Cancer Institute-Sponsored Working Group Guidelines for Chronic Lymphocytic Leukemia: Revised Guidelines for Diagnosis and Treatment. Blood, 87, 4990-4997.

[18] Zenz, T., Hling, S.F., Mertens, D., Dohner, H. and Stilgenbauer, S. (2010) Moving from Prognostic to Predictive Factors in Chronic Lymphocytic Leukaemia (CLL). Best Practice Research: Clinical Haematology Home, 23, 71-84. https://doi.org/10.1016/j.beha.2009.12.003

[19] Hochenbery, D., Nunez, G., Milliman, C., et al. (1990) BCL-2 Is an Inner Mitochondrial Membrane Protein That Blocks Programmed Cell Death. Nature, 348, $334-$ 336. https://doi.org/10.1038/348334a0

[20] Bissonnette, R.P., Echeverri, F., Mahboubi, A., et al. (1992) Apoptotic Cell Death Induced by c-myc Is Inhibited by bcl-2. Nature, 359, 552-554. https://doi.org/10.1038/359552a0

[21] Opferman, J.T., Letai, A., Beard, C., et al. (2003) Development and Maintenance of B and T Lymphocytes Requires Antiapoptotic MCL-1. Nature, 426, 671-676. https://doi.org/10.1038/nature02067

[22] Kitada, S., Andersen, J., Akar, S., Zapata, J.M., Takayama, S., Krajewski, S., et al. (1998) Expression of Apoptosis-Regulating Proteins in Chronic Lymphocytic Leukemia: Correlations with in Vitro and in Vivo Chemoresponses. Blood, 91, 33793389.

[23] Lomo, J., Smeland, E.B., Krajewski, S., Reed, J.C. and Blomhoff, H.K. (1996) Expression of the BCL-2 Homologue MCL-1 Correlates with Survival of Peripheral Blood B Lymphocytes. Cancer Research, 56, 40-43.

[24] Moshynska, O., Sheridan, D.P., Kanthan, R. and Saxena, A. (2002) MCL-1 Gene Nucleotide Replacements in B-Cell Chronic Lymphocytic Leukemia. Laboratory Investigation, 82, 256A (abstract 1066). 
[25] Marschitz, I., Tinhofer, I., Hittmair, A., et al. (2000) Analysis of BCL-2 Protein Expression in Chronic Lymphocytic Leukemia. A Comparison of Three Semiquantitation Techniques. American Journal of Clinical Pathology, 113, 219-229. https://doi.org/10.1309/491W-L1TN-UFQX-T61B

[26] Kitada, S., Anderson, J., Akar, S., et al. (1998) Expression of Apoptosis-Regulating Proteins in Chronic Lymphocytic Leukemia: Correlations with in Vitro and in Vivo Chemoresponses. Blood, 91, 3379-3389.

[27] Lazaridou, A., Miraxtsi, C., Korantzis, J., et al. (2000) Simultaneous Detection of BCL-2 Protein, Trisomy 12, Retinoblastoma and P53 Monoallelic Gene Deletions in B-Cell Chronic Lymphocytic Leukemia by Fluorescence in Situ Hybridization (FISH): Relation to Disease Status. Leukemia \& Lymphoma, 36, 503-512. https://doi.org/10.3109/10428190009148398

[28] Mendez, P., Vargas, A., Bueno, C., et al. (2004) Quantitative Analysis of BCL-2 Expression in Normal and Leukemic Human B-Cell Differentiation. Leukemia, 18, 491-198.

[29] Schimmer, A.D., Munk-Pederson, I., Minden, M.D., et al. (2003) BCL-2 and Apoptosis in Chronic Lymphocytic Leukemia. Current Treatment Options in Oncology, 4, 211-218. https://doi.org/10.1007/s11864-003-0022-y

[30] Del Poeta, G., Del Principe, M.I., Maurillo, L., et al. (2010) Spontaneous Apoptosis and Proliferation Detected by BCL-2 and CD71 Proteins Are Important Progression Indicators within ZAP-70 Negative Chronic Lymphocytic Leukemia. Leukemia \& Lymphoma, 51, 95-106. https://doi.org/10.3109/10428190903350421

[31] Damle, R.N., Wasil, T., Fais, F., et al. (1999) Ig V Gene Mutation Status and CD38 Expression as Novel Prognostic Indicators in Chronic Lymphocytic Leukemia. Blood, 94, 1840-1847.

[32] Pepper, C., Thomas, A., Hoy, T. and Bentley, P. (1999) Chlorambucil Resistance in B-Cell Chronic Lymphocytic Leukaemia Is Mediated through Failed Bax Induction and Selection of High BCL-2-Expressing Subclones. British Journal of Haematology, 104, 581-588. https://doi.org/10.1046/j.1365-2141.1999.01210.x

[33] Klein, A., Miera, O., Bauer, O., Golfier, S. and Schriever, F. (2000) Chemosensitivity of B Cell Chronic Lymphocytic Leukemia and Correlated Expression of Proteins Regulating Apoptosis, Cell Cycle and DNA Repair. Leukemia, 14, 40-46. https://doi.org/10.1038/sj.leu.2401636

[34] Thomas, A., Pepper, C., Hoy, T. and Bentley, P. (2000) BCL-2 and Bax Expression and Chlorambucil-Induced Apoptosis in the T Cells and Leukaemic B-Cells of Untreated B-Cell Chronic Lymphocytic Leukaemia Patients. Leukemia Research, 24, 813-821. https://doi.org/10.1016/S0145-2126(00)00051-5

[35] Stoetzer, O.J., Pogrebniak, A., Scholz, M., Pelka-Fleischer, R., Gullis, E., Darsow, M., et al. (1999) Drug-Induced Apoptosis in Chronic Lymphocytic Leukemia. Leukemia, 13, 1873-1880. https://doi.org/10.1038/sj.leu.2401572

[36] Zaja, F., Di Loreto, C., Amoroso, V., Salmaso, F., Russo, D., Silvestri, F., et al. (1998) BCL-2 Immunohistochemical Evaluation in B-Cell Chronic Lymphocytic Leukemia and Hairy Cell Leukemia before Treatment with Fludarabine and 2-Chlorodeoxyadenosine. Leukemia \& Lymphoma, 28, 567-572.

https://doi.org/10.3109/10428199809058365 
Submit or recommend next manuscript to SCIRP and we will provide best service for you:

Accepting pre-submission inquiries through Email, Facebook, LinkedIn, Twitter, etc. A wide selection of journals (inclusive of 9 subjects, more than 200 journals)

Providing 24-hour high-quality service

User-friendly online submission system

Fair and swift peer-review system

Efficient typesetting and proofreading procedure

Display of the result of downloads and visits, as well as the number of cited articles Maximum dissemination of your research work

Submit your manuscript at: http://papersubmission.scirp.org/

Or contact jet@scirp.org 